\title{
Lower bounds on the moduli of three-dimensional Coulomb-Dirac operators via fractional Laplacians with applications
}

\author{
Sergey Morozov*†and David Müller*
}

\begin{abstract}
For $\nu \in[0,1]$ let $D^{\nu}$ be the distinguished self-adjoint realisation of the three-dimensional Coulomb-Dirac operator $-\mathrm{i} \boldsymbol{\alpha} \cdot \nabla-\nu|\cdot|^{-1}$. For $\nu \in[0,1)$ we prove the lower bound of the form $\left|D^{\nu}\right| \geqslant C_{\nu} \sqrt{-\Delta}$, where $C_{\nu}$ is found explicitly and is better then in all previous works on the topic. In the critical case $\nu=1$ we prove that for every $\lambda \in[0,1)$ there exists $K_{\lambda}>0$ such that the estimate $\left|D^{1}\right| \geqslant K_{\lambda} a^{\lambda-1}(-\Delta)^{\lambda / 2}-a^{-1}$ holds for all $a>0$. As applications we extend the range of coupling constants in the proof of the stability of the relativistic electron-positron field and obtain Cwickel-Lieb-Rozenblum and Lieb-Thirring type estimates on the negative eigenvalues of perturbed projected massless Coulomb-Dirac operators in the Furry picture. We also study the existence of a virtual level at zero for such projected operators.
\end{abstract}

\section{Introduction and main results}

This work is dedicated to the study of the Coulomb-Dirac operator

$$
-\mathrm{i} \boldsymbol{\alpha} \cdot \nabla+M \beta-\nu|\cdot|^{-1}
$$

in $\mathrm{L}^{2}\left(\mathbb{R}^{3}, \mathbb{C}^{4}\right)$. Here $\boldsymbol{\alpha}:=\left(\alpha_{1}, \alpha_{2}, \alpha_{3}\right)$ is the vector of $\alpha_{i}:=\left(\begin{array}{cc}0_{\mathbb{C}^{2}} & \sigma_{i} \\ \sigma_{i} & 0_{\mathbb{C}^{2}}\end{array}\right)$ for $i \in$ $\{1,2,3\}$ with $\sigma_{1}, \sigma_{2}, \sigma_{3}$ being the standard Pauli matrices, $\beta:=\operatorname{diag}(1,1,-1,-1)$ and $M \geqslant 0$ is the mass of the Dirac particle. It is well known that the operator 1.1 is essentially self-adjoint on $C_{0}^{\infty}\left(\mathbb{R}^{3} \backslash\{0\}, \mathbb{C}^{4}\right)$ only for $|\nu| \leqslant \sqrt{3} / 2$. For $\nu \in$ $(\sqrt{3} / 2,1]$ there is a canonical choice of a self-adjoint extension, which for $M=1$ we denote by $D^{\nu, 1}$, see [7, 14] and Remark [2.8 For general $M \geqslant 0$ we let $D^{\nu, M}:=$ $D^{\nu, 1}+(M-1) \beta$. We will restrict our attention to $\nu \in[0,1]$ and the distinguished self-adjoint realisation $D^{\nu, M}$. A special role is played by the homogeneous operator $D^{\nu}:=D^{\nu, 0}$.

\footnotetext{
*Mathematisches Institut, Ludwig-Maximilians-Universität München, Theresienstr. 39, 80333 Munich, Germany

morozov@math.lmu.de,dmueller@math.lmu.de

${ }^{\dagger}$ St. Petersburg State University, Universitetskaya nab. 7-9, 199034 St. Petersburg, Russia
} 
For $\nu \in[0,1]$ we introduce

$$
\Upsilon_{\nu}:=\sqrt{1-\nu^{2}}
$$

We now state the results of the paper. Some of them are related to the corresponding results in two dimensions, which we have obtained in [12]. It turns out that the three-dimensional situation is somewhat simpler due to presence of the Hardy inequality. The key results are Theorems 1.1 and 1.3 . most of the rest are their applications. It is important to note that we provide the explicit constant $C_{\nu}$ in Theorem 1.1, whereas in the corresponding Theorem 1 in [12] substantial work is still required to extract an explicit value from the proof.

In the following scalar operators like $\sqrt{-\Delta}$ are applied to vector-valued functions component-wise without reflecting this in the notation.

\subsection{Lower bounds via powers of the Laplacian}

Theorem 1.1. For every $\nu \in[0,1)$ the inequality

$$
\left|D^{\nu}\right| \geqslant C_{\nu} \sqrt{-\Delta}
$$

holds with

$$
C_{\nu}:=\left(1-\pi \Upsilon_{\nu} \cot \left(\pi \Upsilon_{\nu} / 2\right) / 2\right) \eta_{\nu}
$$

where

$$
\eta_{\nu}:= \begin{cases}\frac{\left(9+4 \nu^{2}\right)^{1 / 2}-4 \nu}{3\left(1-2 \Upsilon_{\nu} \cot \left(\pi \Upsilon_{\nu} / 2\right)\right)}, & \text { for } \nu \in[0,1) \backslash\{\sqrt{3} / 2\} ; \\ \frac{1}{\sqrt{3}(\pi-2)}, & \text { for } \nu=\sqrt{3} / 2 ; \\ \frac{\pi(4-\sqrt{13})}{3(4-\pi)}, & \text { for } \nu=1 .\end{cases}
$$

The following figure shows the graph of $C_{\nu}$ as a function of $\nu$ with the dotted affine function for reference.

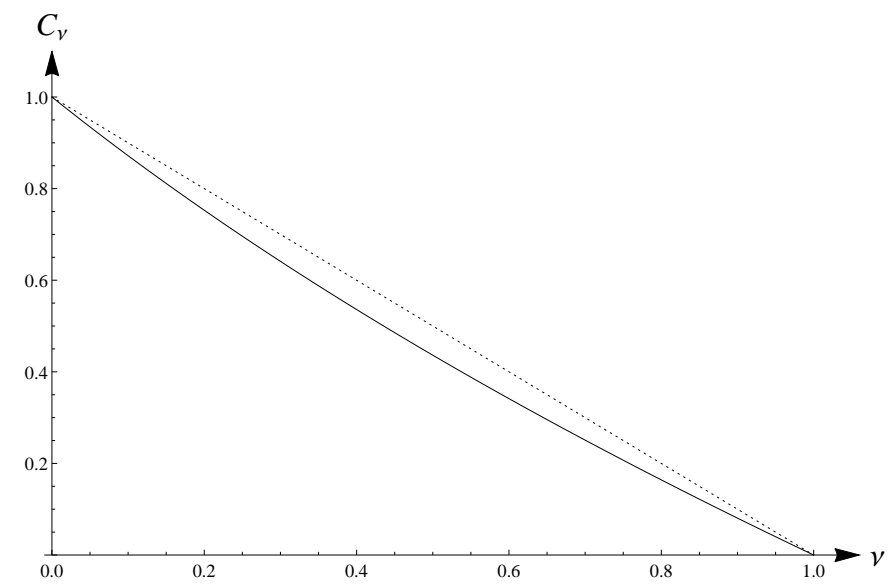


We conclude the following estimates for massive Coulomb-Dirac operators:

Corollary 1.2. For every $\nu \in[0,1)$ and $M>0$ the inequalities

$$
\left|D^{\nu, M}\right| \geqslant \Upsilon_{\nu} C_{\nu} \sqrt{-\Delta}
$$

and

$$
\left|D^{\nu, M}\right| \geqslant \max \left\{\frac{\Upsilon_{\nu} C_{\nu}}{1+C_{\nu}}, 1-2 \nu\right\} \sqrt{-\Delta+M^{2}}
$$

hold with $C_{\nu}$ as in (1.4).

Note that $\left|D^{0, M}\right|=\sqrt{-\Delta+M^{2}}$ holds. The results analogous to Theorem 1.1 and Corollary 1.2 were already discussed in the literature (see e.g. Lemma 1 in 3 ] and Lemma 1 in [6]). However, they were obtained by taking the square root of the inequality

$$
\left(D^{\nu, M}\right)^{2} \geqslant \widetilde{C}_{\nu, M}(-\Delta),
$$

which cannot be valid with $\widetilde{C}_{\nu, M}>0$ for $\nu>\sqrt{3} / 2$, since for such $\nu$ the domain of $D^{\nu}$ is not contained in $\mathrm{H}^{1}\left(\mathbb{R}^{3}, \mathbb{C}^{4}\right)$, see e.g. Lemma 2.9 below. Even for $\nu \in[0, \sqrt{3} / 2]$ the values of the constants in Theorem 1.1 and Corollary 1.2 are improvements upon the above results.

Theorem 1.1 cannot hold for $\nu=1$, which is a consequence of the fact that the domain of $D^{1}$ is not contained in $\mathrm{H}^{1 / 2}\left(\mathbb{R}^{3}, \mathbb{C}^{4}\right)$, see Lemma 2.9. However, we can compare $\left|D^{1}\right|$ with the powers of the Laplacian smaller than $1 / 2$. The comparison is based on the fact that for any $\lambda \in[0,1)$ there exists $L_{\lambda}>0$ such that for all $a>0$ the inequality

$$
(-\Delta)^{1 / 2}-\frac{2}{\pi}|\cdot|^{-1} \geqslant L_{\lambda} a^{\lambda-1}(-\Delta)^{\lambda / 2}-a^{-1}
$$

holds, see Inequality (1.8) of [8] and Theorem 2.3 in [17].

Theorem 1.3. For any $\lambda \in[0,1)$ and $a>0$ the inequality

$$
\left|D^{1}\right| \geqslant K_{\lambda} a^{\lambda-1}(-\Delta)^{\lambda / 2}-a^{-1}
$$

holds with

$$
K_{\lambda}:=\min \left\{L_{\lambda}\left(\frac{\pi(4-\sqrt{13})}{3(4-\pi)}\right)^{\lambda}, \lambda^{-\lambda}(1-\lambda)^{-(1-\lambda)}\left(\frac{\sqrt{229}-8}{15}\right)^{\lambda}\right\}
$$

where $L_{\lambda}$ is as in (1.8). 


\subsection{Stability of the electron-positron field}

Let

$$
\mathbb{H}:=\int \mathrm{d} x: \Psi^{*}(x) D^{\nu, M} \Psi(x):+\frac{\alpha}{2} \int \mathrm{d} x \int \mathrm{d} y \frac{: \Psi^{*}(x) \Psi^{*}(y) \Psi(y) \Psi(x):}{|\mathbf{x}-\mathbf{y}|}
$$

be the Hamiltonian of the electron-positron field in the Furry picture, see 6, 3]. Here $\Psi$ are the field operators and $\alpha>0$ is the fine-structure constant. As explained in Section 2 of [6] (Note the misprint: the inequality in Section 2(ii) of [6] should coincide with inequality (1) there!), estimate (1.6) immediately implies the following result.

Theorem 1.4. Let $\nu \in[0,1), M, \alpha \geqslant 0$. Then for

$$
\alpha \leqslant 4 \Upsilon_{\nu} C_{\nu} / \pi
$$

the energy $\mathcal{E}(\rho):=\rho(\mathbb{H})$ is non-negative for all generalised Hartree-Fock states $\rho$.

For the physical value of the fine-structure constant $\alpha \approx 1 / 137$ condition (1.9) is fulfilled for $\nu \lesssim 0.97$, i.e. for the atomic numbers $Z=\nu / \alpha$ up to and including 132. In Theorem 1 of [6] (which is an improvement upon Theorem 2 in [3]) the same result as in Theorem 1.4 was proved under the stronger assumption

$$
\alpha \leqslant(4 / \pi)\left(1-\nu^{2}\right)^{1 / 2}\left(\sqrt{4 \nu^{2}+9}-4 \nu\right) / 3
$$

instead of (1.9), which for $\alpha \approx 1 / 137$ corresponds to the atomic numbers $Z=$ $\nu / \alpha \leqslant 117$ and cannot hold for any $\alpha>0$ if $\nu>\sqrt{3} / 2$.

\subsection{Eigenvalue estimates in the Furry picture}

Theorems 1.1 and 1.3 allow us to estimate the negative spectrum of perturbed projected massless Coulomb-Dirac operators in the Furry picture (cf. [12]). Namely, for $\nu \in[0,1]$ let $P_{+}^{\nu}$ be the spectral projector of $D^{\nu}$ to the half-line $[0, \infty)$. Assuming that the negative energy states of $D^{\nu}$ are occupied (Dirac sea), we restrict the operator to the Hilbert space $\mathfrak{H}_{+}^{\nu}:=P_{+}^{\nu} \mathrm{L}^{2}\left(\mathbb{R}^{3}, \mathbb{C}^{4}\right)$. We now consider perturbations of such restricted operator by electromagnetic potentials. The following results are three-dimensional equivalents of Corollary 2 and Theorems 3 and 4 in [12. For numbers and self-adjoint operators we use the notation $x_{ \pm}:=\max \{ \pm x, 0\}$ for the positive and negative parts of $x$.

Lemma 1.5. Suppose that $(\nu, \gamma) \in([0,1] \times[0, \infty)) \backslash\{(1,0)\}$. Let $V$ be a measurable Hermitian $(4 \times 4)$-matrix function with $\operatorname{tr}\left(V_{+}^{3+\gamma}\right) \in \mathrm{L}^{1}\left(\mathbb{R}^{3}\right)$ and such that there exists $C>0$ with

$$
\int_{\mathbb{R}^{3}}\left\langle v(\mathbf{x}), V_{-}(\mathbf{x}) v(\mathbf{x})\right\rangle \mathrm{d} \mathbf{x} \leqslant C\left(\left\|\left|D^{\nu}\right|^{1 / 2} v\right\|^{2}+\|v\|^{2}\right), \text { for all } v \in P_{+}^{\nu} \mathfrak{D}\left(\left|D^{\nu}\right|^{1 / 2}\right) .
$$

Then the quadratic form

$$
\begin{aligned}
& \mathfrak{d}^{\nu}(V): P_{+}^{\nu} \mathfrak{D}\left(\left|D^{\nu}\right|^{1 / 2}\right) \rightarrow \mathbb{R}, \\
& \mathfrak{d}^{\nu}(V)[v]:=\left\|\left|D^{\nu}\right|^{1 / 2} v\right\|^{2}-\int_{\mathbb{R}^{3}}\langle v(\mathbf{x}), V(\mathbf{x}) v(\mathbf{x})\rangle \mathrm{d} \mathbf{x}
\end{aligned}
$$


is closed and bounded from below in $\mathfrak{H}_{+}^{\nu}$.

According to Theorem 10.1.2 in [5], there exists a unique self-adjoint operator $D^{\nu}(V)$ in $\mathfrak{H}_{+}^{\nu}$ associated to $\mathfrak{d}^{\nu}(V)$.

In the following theorem we estimate the negative spectrum of $D^{\nu}(V)$ by combining Theorems 1.1 and 1.3 with the estimates

$$
\operatorname{rank}\left((-\Delta)^{t}-V\right)_{-} \leqslant C_{t}^{\Delta} \int_{\mathbb{R}^{3}} \operatorname{tr}\left(V_{+}(\mathbf{x})\right)^{3 /(2 t)} \mathrm{d} \mathbf{x},
$$

which according to Example 3.3 of [9] hold for all $0<t<3 / 2$ with (non-optimal)

$$
C_{t}^{\Delta}:=\frac{1}{4 \pi^{2} t}\left(\frac{3}{3-2 t}\right)^{(3-t) / t} .
$$

We obtain the following estimates on the negative eigenvalues of $D^{\nu}(V)$.

Theorem 1.6. For $\nu, \lambda \in[0,1)$ let $C_{\nu}$ and $K_{\lambda}$ be defined in Theorems 1.1 and 1.3. respectively.

(a) Cwickel-Lieb-Rozenblum inequalities: For $\nu \in[0,1)$,

$$
\operatorname{rank}\left(D^{\nu}(V)\right)_{-} \leqslant C_{1 / 2}^{\Delta} C_{\nu}^{-3} \int_{\mathbb{R}^{3}} \operatorname{tr}\left(V_{+}(\mathbf{x})\right)^{3} \mathrm{~d} \mathbf{x} .
$$

(b) (Hardy-)Lieb-Thirring inequalities: For $\nu \in[0,1]$ and $\gamma>0$, the estimate

$$
\operatorname{tr}\left(D^{\nu}(V)\right)_{-}^{\gamma} \leqslant C_{\nu, \gamma}^{\mathrm{LT}} \int_{\mathbb{R}^{3}} \operatorname{tr}\left(V_{+}(\mathbf{x})\right)^{3+\gamma} \mathrm{d} \mathbf{x}
$$

holds with

$$
\begin{aligned}
C_{\nu, \gamma}^{\mathrm{LT}} & :=\frac{6 C_{1 / 2}^{\Delta} C_{\nu}^{-3}}{(\gamma+1)(\gamma+2)(\gamma+3)}, \quad \text { for } \nu<1 ; \\
C_{1, \gamma}^{\mathrm{LT}} & :=\min _{\lambda \in(3 /(3+\gamma), 1)} \frac{\gamma^{1+\gamma} \lambda^{\gamma} \Gamma(1+3 / \lambda) \Gamma(3+\gamma-3 / \lambda) C_{\lambda / 2}^{\Delta} K_{\lambda}^{-3 / \lambda}}{\Gamma(4+\gamma)(3-3 \lambda)^{3(1-\lambda) / \lambda}((3+\gamma) \lambda-3)^{3+\gamma-3 / \lambda}} .
\end{aligned}
$$

It turns out that (1.10) cannot hold for $\nu=1$ for any constant in front of the integral, since $D^{\nu}(0)$ has a virtual level at zero. This is the result of the theorem below.

Theorem 1.7. Suppose that $V=\left(\begin{array}{cc}V_{\mathrm{I}, \mathrm{I}} & V_{\mathrm{I}, \mathrm{II}} \\ V_{\mathrm{II}, \mathrm{I}} & V_{\mathrm{II}, \mathrm{II}}\end{array}\right)$ is a block matrix function on $\mathbb{R}^{3}$ with $2 \times 2$ blocks which satisfies the assumptions of Lemma 1.5. Using the spherical 
coordinates in $\mathbb{R}^{3}$ we define the $2 \times 2$ matrix functions

$$
\begin{aligned}
& A_{(-1,-1)}(\rho, \theta, \phi):=\left(\begin{array}{cc}
\left\langle\left(\begin{array}{l}
0 \\
1
\end{array}\right), V_{\mathrm{I}, \mathrm{I}}\left(\begin{array}{l}
0 \\
1
\end{array}\right)\right\rangle & \left\langle\left(\begin{array}{l}
0 \\
\mathrm{i}
\end{array}\right), V_{\mathrm{I}, \mathrm{II}}\left(\begin{array}{c}
\mathrm{e}^{-\mathrm{i} \phi} \sin \theta \\
-\cos \theta
\end{array}\right)\right\rangle \\
\left\langle\left(\begin{array}{c}
\mathrm{e}^{-\mathrm{i} \phi} \sin \theta \\
-\cos \theta
\end{array}\right), V_{\mathrm{II}, \mathrm{I}}\left(\begin{array}{l}
0 \\
\mathrm{i}
\end{array}\right)\right\rangle & \left\langle\left(\begin{array}{c}
\mathrm{e}^{-\mathrm{i} \phi} \sin \theta \\
-\cos \theta
\end{array}\right), V_{\mathrm{II}, \mathrm{II}}\left(\begin{array}{c}
\mathrm{e}^{-\mathrm{i} \phi} \sin \theta \\
-\cos \theta
\end{array}\right)\right\rangle
\end{array}\right), \\
& A_{(1,-1)}(\rho, \theta, \phi):=\left(\begin{array}{cc}
\left\langle\left(\begin{array}{l}
1 \\
0
\end{array}\right), V_{\mathrm{I}, \mathrm{I}}\left(\begin{array}{l}
1 \\
0
\end{array}\right)\right\rangle & \left\langle\left(\begin{array}{c}
\mathrm{i} \\
0
\end{array}\right), V_{\mathrm{I}, \mathrm{II}}\left(\begin{array}{c}
\cos \theta \\
\mathrm{e}^{\mathrm{i} \phi} \sin \theta
\end{array}\right)\right\rangle \\
\left\langle\left(\begin{array}{c}
\cos \theta \\
\mathrm{e}^{\mathrm{i} \phi} \sin \theta
\end{array}\right), V_{\mathrm{II}, \mathrm{I}}\left(\begin{array}{l}
\mathrm{i} \\
0
\end{array}\right)\right\rangle & \left\langle\left(\begin{array}{c}
\cos \theta \\
\mathrm{e}^{\mathrm{i} \phi} \sin \theta
\end{array}\right), V_{\mathrm{II}, \mathrm{II}}\left(\begin{array}{c}
\cos \theta \\
\mathrm{e}^{\mathrm{i} \phi} \sin \theta
\end{array}\right)\right\rangle
\end{array}\right), \\
& A_{(-1,1)}(\rho, \theta, \phi):=\left(\begin{array}{cc}
\left\langle\left(\begin{array}{c}
\mathrm{e}^{-\mathrm{i} \phi} \sin \theta \\
-\cos \theta
\end{array}\right), V_{\mathrm{I}, \mathrm{I}}\left(\begin{array}{c}
\mathrm{e}^{-\mathrm{i} \phi} \sin \theta \\
-\cos \theta
\end{array}\right)\right\rangle & \left\langle\left(\begin{array}{c}
\mathrm{e}^{-\mathrm{i} \phi} \sin \theta \\
-\cos \theta
\end{array}\right), V_{\mathrm{I}, \mathrm{II}}\left(\begin{array}{c}
0 \\
-\mathrm{i}
\end{array}\right)\right\rangle \\
\left\langle\left(\begin{array}{c}
0 \\
-\mathrm{i}
\end{array}\right), V_{\mathrm{II}, \mathrm{I}}\left(\begin{array}{c}
\mathrm{e}^{-\mathrm{i} \phi} \sin \theta \\
-\cos \theta
\end{array}\right)\right\rangle & \left\langle\left(\begin{array}{c}
0 \\
1
\end{array}\right), V_{\mathrm{II}, \mathrm{II}}\left(\begin{array}{c}
0 \\
1
\end{array}\right)\right\rangle
\end{array}\right) \\
& A_{(1,1)}(\rho, \theta, \phi):=\left(\begin{array}{cc}
\left\langle\left(\begin{array}{c}
\cos \theta \\
\mathrm{e}^{\mathrm{i} \phi} \sin \theta
\end{array}\right), V_{\mathrm{I}, \mathrm{I}}\left(\begin{array}{c}
\cos \theta \\
\mathrm{e}^{\mathrm{i} \phi} \sin \theta
\end{array}\right)\right\rangle & \left\langle\left(\begin{array}{c}
\cos \theta \\
\mathrm{e}^{\mathrm{i} \phi} \sin \theta
\end{array}\right), V_{\mathrm{I}, \mathrm{II}}\left(\begin{array}{c}
-\mathrm{i} \\
0
\end{array}\right)\right\rangle \\
\left\langle\left(\begin{array}{c}
-\mathrm{i} \\
0
\end{array}\right), V_{\mathrm{II}, \mathrm{I}}\left(\begin{array}{c}
\cos \theta \\
\mathrm{e}^{\mathrm{i} \phi} \sin \theta
\end{array}\right)\right\rangle & \left\langle\left(\begin{array}{l}
1 \\
0
\end{array}\right), V_{\mathrm{II}, \mathrm{II}}\left(\begin{array}{l}
1 \\
0
\end{array}\right)\right\rangle
\end{array}\right),
\end{aligned}
$$

where $V_{p, q}$ with $p, q \in\{\mathrm{I}, \mathrm{II}\}$ are taken at $(\rho, \theta, \phi)$. For $\mathbf{j} \in\{-1,1\}^{2}$ and $\rho>0$ let

$$
W_{\mathbf{j}}(\rho):=\int_{0}^{\pi} \int_{0}^{2 \pi} A_{\mathbf{j}}(\rho, \theta, \phi) \mathrm{d} \phi \sin \theta \mathrm{d} \theta .
$$

If for some $\mathbf{j} \in\{-1,1\}^{2}$ we have

$$
\left\|W_{\mathbf{j}}\right\|_{\mathbb{C}^{2 \times 2}} \in \mathrm{L}^{1}\left(\mathbb{R}_{+}, \rho^{2} \mathrm{~d} \rho\right), \quad \int_{0}^{\infty}\left\langle\left(\begin{array}{c}
1 \\
j_{2}
\end{array}\right),\left|W_{\mathbf{j}}(\rho)\right|\left(\begin{array}{c}
1 \\
j_{2}
\end{array}\right)\right\rangle \mathrm{d} \rho<\infty
$$

and

$$
\int_{0}^{\infty}\left\langle\left(\begin{array}{c}
1 \\
j_{2}
\end{array}\right), W_{\mathbf{j}}(\rho)\left(\begin{array}{c}
1 \\
j_{2}
\end{array}\right)\right\rangle \mathrm{d} \rho>0
$$

then the operator $D^{1}(V)$ has at least one negative eigenvalue.

In particular, $D^{1}\left(v \mathbb{I}_{4}\right)$ has negative eigenvalues for any non-zero continuous scalar function $v \geqslant 0$, which quickly decays at infinity.

The article is organised as follows: We do not provide the details of the proofs which are fully analogous to those of [12]. This, in particular, applies to Lemma 1.5 and Theorem 1.6. In Section 2 we prepare useful representations of operators of interest with the help of certain unitary transforms. Section 3 is dedicated to the study of the operator $(-\Delta)^{1 / 2}-\alpha|\cdot|^{-1}$ in the representation, in which it can be relatively easily compared with $\left|D^{\nu}\right|$. Such comparison is done separately in different channels of the angular momentum decomposition in Section 4 . Some less interesting technical parts of the proofs are relegated to the appendices. Finally, in Section [5] we complete the proofs of Theorem 1.1. Corollary [1.2 and Theorems 1.3 and 1.7

Acknowledgement: S. M. was supported by the RSF grant 15-11-30007. 


\section{Mellin, Fourier and related transforms in spher- ical coordinates}

Let $(\rho, \theta, \phi),(\varrho, \vartheta, \varphi) \in[0, \infty) \times[0, \pi) \times[0,2 \pi)$ be the spherical coordinates for $\mathbb{R}^{3}$ in coordinate and momentum spaces, respectively.

Fourier transform. We use the standard unitary Fourier transform in $\mathrm{L}^{2}\left(\mathbb{R}^{3}\right)$ given in the spherical coordinates for $v \in L^{1}\left(\mathbb{R}^{3}\right) \cap L^{2}\left(\mathbb{R}^{3}\right)$ by

$$
\begin{aligned}
(\mathcal{F} v)(\varrho, \vartheta, \varphi):= & \frac{1}{(2 \pi)^{3 / 2}} \int_{0}^{\infty} \int_{0}^{\pi} \int_{0}^{2 \pi} \mathrm{e}^{-\mathrm{i} \varrho \rho(\sin \theta \sin \vartheta \cos (\phi-\varphi)+\cos \theta \cos \vartheta)} \\
& \times v(\rho, \theta, \phi) \mathrm{d} \phi \sin \theta \mathrm{d} \theta \rho^{2} \mathrm{~d} \rho .
\end{aligned}
$$

Let $\mathbb{S}^{2}$ be the unit sphere in $\mathbb{R}^{3}$. As an orthonormal basis in $L^{2}\left(\mathbb{S}^{2} ; \mathbb{C}^{2}\right)$ we use the spherical spinors $\Omega_{l, m, s}$, which are defined by (2.1.25) and (2.1.26) in [4], with $l \in \mathbb{N}_{0}, \quad m \in\{-l-1 / 2, \ldots, l+1 / 2\}$ and $s \in\{-1 / 2,1 / 2\}$. The corresponding index set is denoted by

$$
\mathfrak{T}:=\left\{(l, m, s): l \in \mathbb{N}_{0}, m \in\left\{-l-\frac{1}{2}, \ldots, l+\frac{1}{2}\right\}, s= \pm \frac{1}{2}, \Omega_{l, m, s} \neq 0\right\},
$$

see $(2.1 .27)$ in 4 .

Lemma 2.1. For $(l, m, s) \in \mathfrak{T}$ and $\psi \in \mathrm{C}_{0}^{\infty}([0, \infty))$ the Fourier transform of

$$
\Psi_{l, m, s}(\rho, \theta, \phi):=\rho^{-1} \psi(\rho) \Omega_{l, m, s}(\theta, \phi)
$$

is given in the spherical coordinates by

$$
\left(\mathcal{F} \Psi_{l, m, s}\right)(\varrho, \vartheta, \varphi)=(-\mathrm{i})^{l} \int_{0}^{\infty} \sqrt{\frac{\rho}{\varrho}} J_{l+1 / 2}(\rho \varrho) \psi(\rho) \mathrm{d} \rho \Omega_{l, m, s}(\vartheta, \varphi) .
$$

Here $J_{l+1 / 2}$ is the Bessel function of the first kind.

Proof. According to 10.60.7, 14.7.1, 14.7.17, 14.30.9 and 10.47.3 in [1] the relation

$$
\begin{aligned}
& \exp (-\mathrm{i} \varrho \rho(\sin \theta \sin \vartheta \cos (\phi-\varphi)+\cos \theta \cos \vartheta)) \\
& =(2 \pi)^{3 / 2} \sum_{l=0}^{\infty}(-\mathrm{i})^{l}(\rho \varrho)^{-1 / 2} J_{l+1 / 2}(\rho \varrho) \sum_{m=-l}^{l} \overline{Y_{l, m}(\theta, \phi)} Y_{l, m}(\vartheta, \varphi)
\end{aligned}
$$

holds. Here for $l \in \mathbb{N}_{0}$ and $m \in\{-l,-l+1, \ldots, l-1, l\}$ the functions $Y_{l, m}$ are the spherical harmonics, see 14.30.1 in [1. Substituting (2.2) into (2.1) and using (2.4) together with $(2.1 .25),(2.1 .26)$ in [4] and 14.30.8 in [1] we obtain (2.3). 
Mellin transform. Let $\mathcal{M}$ be the unitary Mellin transform, first defined on $\mathrm{C}_{0}^{\infty}\left(\mathbb{R}_{+}\right)$by

$$
(\mathcal{M} \psi)(\tau):=\frac{1}{\sqrt{2 \pi}} \int_{0}^{\infty} r^{-1 / 2-\mathrm{i} \tau} \psi(r) \mathrm{d} r,
$$

and then extended to a unitary operator $\mathcal{M}: \mathrm{L}^{2}\left(\mathbb{R}_{+}\right) \rightarrow \mathrm{L}^{2}(\mathbb{R})$, see e.g. [11].

Definition 2.2. For $\lambda \in \mathbb{R} \backslash\{0\}$ let $\mathfrak{D}^{\lambda}$ be the set of functions $\psi \in \mathrm{L}^{2}(\mathbb{R})$ such that there exists $\Psi$ analytic in the strip $\mathfrak{S}^{\lambda}:=\{z \in \mathbb{C}: \operatorname{Im} z / \lambda \in(0,1)\}$ with the properties

1. $\underset{t \rightarrow+0}{\mathrm{~L}^{2}-\lim _{t \rightarrow}} \Psi(\cdot+\mathrm{i} t \lambda)=\psi$

2. there exists $\underset{t \rightarrow 1-0}{L^{2}-\lim _{t \rightarrow 0}} \Psi(\cdot+\mathrm{i} t \lambda)$;

$$
\text { 3. } \sup _{t \in(0,1)} \int_{\mathbb{R}}|\Psi(\tau+\mathrm{i} t \lambda)|^{2} \mathrm{~d} \tau<\infty \text {. }
$$

For $\lambda \in \mathbb{R}$ let the operator of multiplication by $r^{\lambda}$ in $\mathrm{L}^{2}\left(\mathbb{R}_{+}, \mathrm{d} r\right)$ be defined on its maximal domain $\mathrm{L}^{2}\left(\mathbb{R}_{+},\left(1+r^{2 \lambda}\right) \mathrm{d} r\right)$. Let $R^{\lambda}: \mathfrak{D}^{\lambda} \rightarrow \mathrm{L}^{2}(\mathbb{R})$ be the linear operator defined by

$$
R^{\lambda} \psi:= \begin{cases}\mathrm{L}^{2}-\lim _{t \rightarrow 1-0} \Psi(\cdot+\mathrm{i} t \lambda), & \lambda \neq 0 \\ \psi, & \lambda=0,\end{cases}
$$

with $\Psi$ as in Definition 2.2. According to (14) in [12],

$$
\mathcal{M} r^{\lambda} \mathcal{M}^{*}=R^{\lambda}
$$

holds for all $\lambda \in \mathbb{R}$ (see also 11, Section II).

The following lemma can be proved in the same way as Lemma 8 in [12].

Lemma 2.3. Let $l \in \mathbb{N}_{0}$. The relation

$$
\left(\mathcal{M}\left((-\mathrm{i})^{l} \int_{0}^{\infty} \sqrt{\cdot r} J_{l+1 / 2}(\cdot r) \psi(r) \mathrm{d} r\right)\right)(\tau)=\Xi_{l}(\tau)(\mathcal{M} \psi)(-\tau)
$$

holds for every $\psi \in C_{0}^{\infty}([0, \infty))$ and $\tau \in \mathbb{R}$ with

$$
\Xi_{l}(\tau):=(-\mathrm{i})^{l} 2^{-\mathrm{i} \tau} \frac{\Gamma((l+3 / 2-\mathrm{i} \tau) / 2)}{\Gamma((l+3 / 2+\mathrm{i} \tau) / 2)} .
$$

Remark 2.4. For any $l \in \mathbb{N}_{0}$ the function $\Xi_{l}$ introduced in (2.7) allows a unique analytic continuation to $\mathbb{C} \backslash\left(-\mathrm{i}\left(l+3 / 2+2 \mathbb{N}_{0}\right)\right)$, whereas

$$
\Xi_{l}^{-1}=\overline{\Xi_{l}(\cdot)}
$$

allows a unique analytic continuation to $\mathbb{C} \backslash\left(\mathrm{i}\left(l+3 / 2+2 \mathbb{N}_{0}\right)\right)$. 
Note that for all $l \in \mathbb{N}_{0}$ the function $\Xi_{l}^{-1}$ has no pole in $\overline{\mathfrak{S}^{1}}$. This situation is different (and simpler) than the one investigated in [12. The reason can be traced back to the non-existence of the Hardy inequality in $\mathbb{R}^{2}$ in opposite to $\mathbb{R}^{3}$. As a consequence, $\psi \in \mathfrak{D}^{1}$ always implies $\Xi_{l}^{-1} \psi \in \mathfrak{D}^{1}$. Thus the assumptions in the following lemma can be relaxed in comparison to Lemma 10 and Corollary 11 in [12. The arguments used in the proofs of these statements can also be successfully applied here.

Lemma 2.5. For $l \in \mathbb{N}_{0}$ and $\psi \in \mathfrak{D}^{1}$ the identity

$$
\Xi_{l} R^{1} \Xi_{l}^{-1} \psi=V_{l}(\cdot+\mathrm{i} / 2) R^{1} \psi
$$

holds with

$$
V_{l}(z):=\frac{\Gamma((l+1+\mathrm{i} z) / 2) \Gamma((l+1-\mathrm{i} z) / 2)}{2 \Gamma((l+2+\mathrm{i} z) / 2) \Gamma((l+2-\mathrm{i} z) / 2)},
$$

for $z \in(\mathbb{C} \backslash \mathrm{i} \mathbb{Z}) \cup\{0\}$.

The following lemma can be proved analogously to Lemma 12 in [12].

Lemma 2.6. For every $l \in \mathbb{N}_{0}$ the function (2.8) is analytic in $(\mathbb{C} \backslash \mathrm{i} \mathbb{Z}) \cup\{0\}$ and has the following properties:

1. $V_{l}(z)=V_{l}(-z)$, for all $z \in(\mathbb{C} \backslash \mathrm{i} \mathbb{Z}) \cup\{0\} ;$

2. $V_{l}(\tau)$ is positive and strictly monotonously decreasing for $\tau \in \mathbb{R}_{+}$;

3. $V_{l}(\mathrm{i} \zeta)$ is positive and strictly monotonously increasing for $\zeta \in[0,1)$;

4. The relation

$$
\left(z^{2}+(l+1)^{2}\right) V_{l}(z)=\left(V_{l+1}(z)\right)^{-1}
$$

holds for all $z \in(\mathbb{C} \backslash \mathrm{i} \mathbb{Z}) \cup\{0\}$.

Angular decomposition. We can represent arbitrary $u \in \mathrm{L}^{2}\left(\mathbb{R}^{3}, \mathbb{C}^{2}\right)$ in the spherical coordinates as

$$
u(\rho, \theta, \phi)=\sum_{(l, m, s) \in \mathfrak{T}} \rho^{-1} u_{l, m, s}(\rho) \Omega_{l, m, s}(\theta, \phi) ;
$$

with

$$
u_{l, m, s}(\rho):=\rho \int_{0}^{2 \pi} \int_{0}^{\pi}\left\langle\Omega_{l, m, s}(\theta, \phi), u(\rho, \theta, \phi)\right\rangle_{\mathbb{C}^{2}} \sin (\theta) \mathrm{d} \theta \mathrm{d} \phi .
$$

The angular momentum decomposition associated to (1.1) is given by

$$
\mathcal{A}: \mathrm{L}^{2}\left(\mathbb{R}^{3}, \mathbb{C}^{4}\right) \rightarrow \bigoplus_{(l, m, s) \in \mathfrak{T}} \mathrm{L}^{2}\left(\mathbb{R}_{+}, \mathbb{C}^{2}\right) ; \quad\left(\begin{array}{l}
\psi_{1} \\
\psi_{2} \\
\psi_{3} \\
\psi_{4}
\end{array}\right) \mapsto \underset{(l, m, s) \in \mathfrak{T}}{\bigoplus}\left(\begin{array}{c}
\left(\begin{array}{l}
\psi_{1} \\
\psi_{2}
\end{array}\right)_{l, m, s} \\
\mathrm{i}\left(\begin{array}{l}
\psi_{3} \\
\psi_{4}
\end{array}\right)_{l+2 s, m,-s}
\end{array}\right)
$$


Let $\nu \in[0,1]$. For $l \in \mathbb{N}_{0}$ and $s \in\{-1 / 2,1 / 2\}$ we define the operators $\widetilde{D}_{l, s}^{\nu}$ in $L^{2}\left(\mathbb{R}_{+}, \mathbb{C}^{2}\right)$ by the differential expressions

$$
\left(\begin{array}{cc}
\frac{-\frac{\nu}{r}}{\mathrm{~d} r}-\frac{2 s l+s+1 / 2}{r} & -\frac{\mathrm{d}}{\mathrm{d} r}-\frac{2 s l+s+1 / 2}{r} \\
-\frac{\nu}{r}
\end{array}\right)
$$

on $\mathrm{C}_{0}^{\infty}\left(\mathbb{R}_{+}, \mathbb{C}^{2}\right)$. Furthermore, we introduce $\widetilde{D}^{\nu}$ as the operator corresponding to (1.1) on the domain $C_{0}^{\infty}\left(\mathbb{R}^{3} \backslash\{0\}, \mathbb{C}^{4}\right)$.

The next lemma follows from Section 2.1 in [4].

Lemma 2.7. For $\nu \in[0,1]$ the operator $\left(\widetilde{D}^{\nu}\right)^{*}$ satisfies

$$
\mathcal{A}\left(\widetilde{D}^{\nu}\right)^{*} \mathcal{A}^{*}=\bigoplus_{(l, m, s) \in \mathfrak{T}}\left(\widetilde{D}_{l, s}^{\nu}\right)^{*} .
$$

Remark 2.8. By Theorem 4 in 77 there exists a unique self-adjoint extension $D^{\nu, 1}$ of $\widetilde{D}^{\nu}+\beta$ which has a positive Schur complement for $\nu \in[0,1]$. Moreover, for $\nu \in[0,1)$ the operator $D^{\nu}:=D^{\nu, 1}-\beta$ is the only self-adjoint extension of $\widetilde{D}^{\nu}$ with the property that every function in its domain possesses finite kinetic energy, i.e. belongs to $\mathrm{H}^{1 / 2}\left(\mathbb{R}^{3}, \mathbb{C}^{4}\right)$ (see Corollary 6 in [14]).

The following property of $D^{\nu}$ follows from Theorem 5 in [14.

Lemma 2.9. Let $\nu \in[0,1]$. The set

$$
\mathfrak{C}^{\nu}:=\mathbb{C}_{0}^{\infty}\left(\mathbb{R}^{3} \backslash\{0\}, \mathbb{C}^{4}\right) \dot{+} \begin{cases}\{0\}, & \text { if } \nu \in[0, \sqrt{3} / 2] ; \\ \operatorname{span}\left\{\Psi_{\mathbf{k}}^{\nu}: \mathbf{k} \in\{-1 / 2,1 / 2\}^{2}\right\}, & \text { otherwise }\end{cases}
$$

with

$$
\Psi_{\mathbf{k}}^{\nu}(\rho, \theta, \phi):=\sqrt{2 \pi} \mathrm{e}^{-\rho} \rho^{\Upsilon_{\nu}-1}\left(\begin{array}{c}
\nu \Omega_{\frac{1}{2}+k_{2}, k_{1},-k_{2}}(\theta, \phi) \\
-\mathrm{i}\left(\Upsilon_{\nu}+(-1)^{\frac{1}{2}-k_{2}}\right) \Omega_{\frac{1}{2}-k_{2}, k_{1}, k_{2}}(\theta, \phi)
\end{array}\right)
$$

is an operator core for $D^{\nu}$.

Proof. It is known that $C_{0}^{\infty}\left(\mathbb{R}^{3} \backslash\{0\}, \mathbb{C}^{4}\right)$ is dense in $\mathrm{H}^{1}\left(\mathbb{R}^{3}, \mathbb{C}^{4}\right)$, combine e.g. Theorem 3.23 and Example 5.26 in [2] with Proposition 9 of Appendix A in [16]. By the Hardy inequality the graph norm of $\widetilde{D}^{\nu}$ is subordinate to the norm of $\mathrm{H}^{1}\left(\mathbb{R}^{3}, \mathbb{C}^{4}\right)$. Hence the closure of $\mathrm{C}_{0}^{\infty}\left(\mathbb{R}^{3} \backslash\{0\}, \mathbb{C}^{4}\right)$ with respect to the graph norm of $D^{\nu}$ contains $\mathrm{H}^{1}\left(\mathbb{R}^{3}, \mathbb{C}^{4}\right)$.

By Theorem 5 in 14, (2.15) would be an operator core for $D^{\nu}$ if we replace $\mathrm{e}^{-\rho}$ in (2.13) by $\xi(\rho)$, where $\xi \in \mathrm{C}^{\infty}\left(\mathbb{R}_{+},[0,1]\right)$ satisfies $\xi(r)=1$ for $r \in(0,1)$ and $\xi(r)=0$ for $r>2$.

Now it is enough to establish that for $\mathbf{k} \in\{-1 / 2,1 / 2\}^{2}$ the functions

$$
\Phi_{\mathbf{k}}^{\nu}(\rho, \theta, \phi):=\left(\mathrm{e}^{-\rho}-\xi(\rho)\right) \rho^{\Upsilon_{\nu}-1}\left(\begin{array}{c}
\nu \Omega_{\frac{1}{2}+k_{2}, k_{1},-k_{2}}(\theta, \phi) \\
-\mathrm{i}\left(\Upsilon_{\nu}+(-1)^{\frac{1}{2}-k_{2}}\right) \Omega_{\frac{1}{2}-k_{2}, k_{1}, k_{2}}(\theta, \phi)
\end{array}\right)
$$


belong to $\mathrm{H}^{1}\left(\mathbb{R}^{3}, \mathbb{C}^{4}\right)$. To do this we observe that by Lemma 2.7

$$
\left\|\nabla \Phi_{\mathbf{k}}^{\nu}\right\|^{2}=\left\|D^{0} \Phi_{\mathbf{k}}^{\nu}\right\|^{2}=\left\|D_{\frac{1}{2}+k_{2}, k_{1},-k_{2}}^{0}\left(\mathrm{e}^{-\rho}-\xi(\rho)\right) \rho^{\Upsilon_{\nu}}\left(\begin{array}{c}
\nu \\
\Upsilon_{\nu}+(-1)^{\frac{1}{2}-k_{2}}
\end{array}\right)\right\|^{2}
$$

holds, where the finiteness of the right hand side follows by a straightforward calculation based on (2.12).

Let $(l, m, s) \in \mathfrak{T}$ and $\nu \in[0,1]$. Lemma 2.7 implies that there is a unique self-adjoint extension $D_{l, s}^{\nu}$ of $\widetilde{D}_{l, s}^{\nu}$ such that

$$
D_{l, s}^{\nu}=\left(\mathcal{A} D^{\nu} \mathcal{A}^{*}\right)_{l, m, s}
$$

holds. By Lemma 2.9 we conclude that

$$
\mathfrak{C}_{l, s}^{\nu}:=\mathrm{C}_{0}^{\infty}\left(\mathbb{R}_{+}, \mathbb{C}^{2}\right) \dot{+} \begin{cases}\operatorname{span}\left\{\psi_{s}^{\nu}\right\}, & \text { if } \nu \in(\sqrt{3} / 2,1] \text { and } l=0, s=1 / 2 \\ \{0\}, & \text { or } l=1, s=-1 / 2 ; \\ \text { otherwise, }\end{cases}
$$

with

$$
\psi_{s}^{\nu}(\rho):=\sqrt{2 \pi} \mathrm{e}^{-\rho} \rho^{\Upsilon_{\nu}}\left(\begin{array}{c}
\nu \\
\Upsilon_{\nu}+(-1)^{\frac{1}{2}+s}
\end{array}\right) \text { for } \rho \in \mathbb{R}_{+}
$$

is an operator core for $D_{l, s}^{\nu}$.

MWF-transform. We now introduce the unitary transform

$$
\mathcal{T}: \mathrm{L}^{2}\left(\mathbb{R}^{3}, \mathbb{C}^{4}\right) \rightarrow \bigoplus_{(l, m, s) \in \mathfrak{T}} \mathrm{L}^{2}\left(\mathbb{R}, \mathbb{C}^{2}\right), \quad \mathcal{T}:=\mathcal{M} \mathcal{A} \operatorname{diag}(1,1,-\mathrm{i},-\mathrm{i}) \mathcal{F},
$$

where $\mathcal{M}$ acts fibre-wise. A direct calculation using Lemmata 2.1 and 2.3 gives

$$
\mathcal{T}\left(\begin{array}{l}
v_{1} \\
v_{2} \\
v_{3} \\
v_{4}
\end{array}\right)=\bigoplus_{(l, m, s) \in \mathfrak{T}}\left(\begin{array}{c}
\mathcal{T}_{l}\left(\begin{array}{l}
v_{1} \\
v_{2}
\end{array}\right)_{l, m, s} \\
\mathcal{T}_{l+2 s}\left(\begin{array}{l}
v_{3} \\
v_{4}
\end{array}\right)_{l+2 s, m,-s}
\end{array}\right),
$$

where for $(l, m, s) \in \mathfrak{T}$ the operators $\mathcal{T}_{l}: \mathrm{L}^{2}\left(\mathbb{R}_{+}\right) \rightarrow \mathrm{L}^{2}(\mathbb{R})$ are given by

$$
\left(\mathcal{T}_{l} \psi\right)(\tau):=\Xi_{l}(\tau)(\mathcal{M} \psi)(-\tau) \text { for any } \psi \in \mathrm{L}^{2}\left(\mathbb{R}_{+}\right) .
$$

In the following two lemmata we study the actions of several operators in the MWF-representation.

Lemma 2.10. The relations

$$
\mathcal{T}(-\mathrm{i} \boldsymbol{\alpha} \cdot \nabla) \mathcal{T}^{*}=\bigoplus_{(l, m, s) \in \mathfrak{T}}\left(R^{1} \otimes \sigma_{1}\right)
$$


and for any $\lambda \in \mathbb{R}$

$$
\mathcal{T}\left((-\Delta)^{\lambda / 2} \otimes \mathbb{1}_{\mathbb{C}^{4}}\right) \mathcal{T}^{*}=\bigoplus_{(l, m, s) \in \mathfrak{T}} R^{\lambda} \otimes \mathbb{1}_{\mathbb{C}^{2}}
$$

hold.

Lemma 2.10 follows immediately by Lemma 8 in [14] and (2.6). The next lemma can be proved as Lemma 19 in [12].

Lemma 2.11. The relation

$$
\mathcal{T}\left(|\cdot|^{-1} \otimes \mathbb{1}_{\mathbb{C}^{4}}\right) \mathcal{T}^{*}=\bigoplus_{(l, m, s) \in \mathfrak{T}}\left(\begin{array}{cc}
\Xi_{l} R^{1} \Xi_{l}^{-1} & 0 \\
0 & \Xi_{l+2 s} R^{1} \Xi_{l+2 s}^{-1}
\end{array}\right)
$$

holds.

U-transform. Let $(l, m, s) \in \mathfrak{T}$. We define the following unitary operator

$$
\mathcal{U}_{l, s}: \mathrm{L}^{2}\left(\mathbb{R}_{+}, \mathbb{C}^{2}\right) \rightarrow \mathrm{L}^{2}\left(\mathbb{R}, \mathbb{C}^{2}\right) ; \quad \mathcal{U}_{l, s}\left(\begin{array}{c}
\psi_{1} \\
\psi_{2}
\end{array}\right):=\left(\begin{array}{c}
\mathcal{T}_{l} \psi_{1} \\
-\mathrm{i} \mathcal{T}_{l+2 s} \psi_{2}
\end{array}\right)
$$

Note the relation

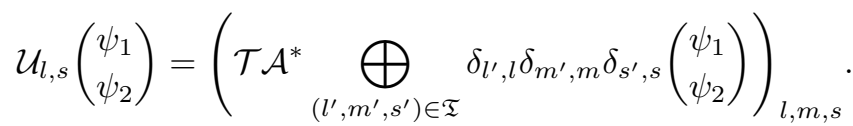

A straightforward calculation involving (2.17), (2.5), (2.8), (2.9) and the elementary properties of the gamma function delivers

Lemma 2.12. Let $\nu \in(\sqrt{3} / 2,1]$. For $s \in\{-1 / 2,1 / 2\}$ the functions (2.16) from the operator core $\mathfrak{C}_{1 / 2-s, s}^{\nu}$ of $D_{1 / 2-s, s}^{\nu}$ satisfy the relation

$$
\mathcal{U}_{1 / 2-s, s} \psi_{s}^{\nu}=\left(\begin{array}{c}
\xi_{s}^{\nu} \\
\eta_{s}^{\nu}
\end{array}\right)+\chi_{s}^{\nu}\left(\begin{array}{c}
1 \\
\nu V_{1 / 2-s}\left(\mathrm{i} \Upsilon_{\nu}\right)
\end{array}\right)
$$

where we have introduced

$$
\begin{aligned}
\chi_{s}^{\nu}(\tau) & :=\nu \Xi_{1 / 2-s}\left(\mathrm{i}\left(\Upsilon_{\nu}+1 / 2\right)\right) \frac{(\tau-\mathrm{i}) \Gamma\left(\mathrm{i} \tau+\Upsilon_{\nu}+1 / 2\right)}{\mathrm{i}\left(\Upsilon_{\nu}-1 / 2\right)} \\
\xi_{s}^{\nu}(\tau) & :=\nu \Gamma\left(\mathrm{i} \tau+\Upsilon_{\nu}+1 / 2\right)\left(\Xi_{1 / 2-s}(\tau)-\frac{(\tau-\mathrm{i}) \Xi_{1 / 2-s}\left(\mathrm{i}\left(\Upsilon_{\nu}+1 / 2\right)\right)}{\mathrm{i}\left(\Upsilon_{\nu}-1 / 2\right)}\right), \\
\eta_{s}^{\nu}(\tau) & :=\mathrm{i}\left(2 s-\Upsilon_{\nu}\right) \Gamma\left(\mathrm{i} \tau+\Upsilon_{\nu}+1 / 2\right)\left(\Xi_{s+1 / 2}(\tau)-\frac{(\tau-\mathrm{i}) \Xi_{s+1 / 2}\left(\mathrm{i}\left(\Upsilon_{\nu}+1 / 2\right)\right)}{\mathrm{i}\left(\Upsilon_{\nu}-1 / 2\right)}\right) .
\end{aligned}
$$




\section{On the Friedrichs extension of the relativistic Coulomb operator in the Fourier-Mellin space}

For $\alpha \in \mathbb{R}$ in $\mathrm{L}^{2}\left(\mathbb{R}^{3}, \mathbb{C}\right)$ consider the symmetric operator

$$
\widetilde{H}^{\alpha}:=(-\Delta)^{1 / 2}-\alpha|\cdot|^{-1}
$$

on the domain $\mathrm{H}^{1}\left(\mathbb{R}^{3}, \mathbb{C}\right)$. Let $\mathfrak{A}:=\left\{(l, m) \in \mathbb{Z}^{2}: l \geqslant 0,-l \leqslant m \leqslant l\right\}$. For every scalar function $u \in \mathrm{L}^{2}\left(\mathbb{R}^{3}, \mathbb{C}\right)$ the decomposition in the spherical harmonics (see Section 14.30 in [1]) is given by

$$
\begin{aligned}
u(\rho, \theta, \phi) & =\sum_{(l, m) \in \mathfrak{A}} \rho^{-1} u_{l, m}(\rho) Y_{l, m}(\theta, \phi), \\
u_{l, m}(\rho) & :=\rho \int_{0}^{2 \pi} \int_{0}^{\pi} \overline{Y_{l, m}(\theta, \phi)} u(\rho, \theta, \phi) \sin \theta \mathrm{d} \theta \mathrm{d} \phi .
\end{aligned}
$$

Introducing the corresponding unitary operator

$$
\mathcal{R}: \mathrm{L}^{2}\left(\mathbb{R}^{3}, \mathbb{C}\right) \rightarrow \bigoplus_{(l, m) \in \mathfrak{A}} \mathrm{L}^{2}\left(\mathbb{R}_{+}, \mathbb{C}\right), \quad \mathcal{R}: u \mapsto \bigoplus_{(l, m) \in \mathfrak{A}} u_{l, m}
$$

we observe the relation (cf. the beginning of Section 3 in [12])

$$
(\mathcal{M R \mathcal { F }}) \widetilde{H}^{\alpha}(\mathcal{M R \mathcal { F }})^{*}=\bigoplus_{(l, m) \in \mathfrak{A}}\left(1-\alpha V_{l}(\cdot+\mathrm{i} / 2)\right) R^{1}=: \bigoplus_{(l, m) \in \mathfrak{A}} \widetilde{H}_{l}^{\alpha}
$$

where the right hand side is an orthogonal sum of operators in $L^{2}(\mathbb{R}, \mathbb{C})$ densely defined on $\mathfrak{D}^{1}$.

In the next lemma, which follows from Lemmata 2.5 and 2.6 in the same way as Lemma 21 in [12], we state the optimal condition on $\alpha$ such that $\widetilde{H}_{l}^{\alpha}$ is bounded from below.

Lemma 3.1. For $l \in \mathbb{N}_{0}$ and $\alpha \in \mathbb{R}$ the operator $\widetilde{H}_{l}^{\alpha}$ is symmetric. It is bounded below (and non-negative) in $\mathrm{L}^{2}(\mathbb{R})$ if and only if

$$
\alpha \leqslant \alpha_{l}:=\frac{1}{V_{l}(0)}=\frac{2 \Gamma^{2}((l+2) / 2)}{\Gamma^{2}((l+1) / 2)} .
$$

Given $l \in \mathbb{N}_{0}$, Lemma 3.1 allows us for $\alpha \leqslant \alpha_{l}$ to pass from the symmetric operator $\widetilde{H}_{l}^{\alpha}$ to the self-adjoint operator $H_{l}^{\alpha}$ by Friedrichs extension [10].

Lemma 3.2. For $\lambda \in(0,1)$ the inequality

$$
H_{0}^{\alpha_{0}} \geqslant L_{\lambda} a^{\lambda-1} R^{\lambda}-a^{-1}
$$

holds for all $a>0$ with $L_{\lambda}$ as in (1.8). 
Proof. For any $v \in \mathfrak{D}^{1}$ we have

$$
\left\langle v, \widetilde{H}_{0}^{\alpha_{0}} v\right\rangle=\left\langle v, R^{1} v\right\rangle-\alpha_{0}\left\langle v, V_{0}(\cdot+\mathrm{i} / 2) R^{1} v\right\rangle .
$$

Letting

$$
\Phi:=(\mathcal{M R \mathcal { F }})^{*} \bigoplus_{(l, m) \in \mathfrak{A}} \delta_{l, 0} \delta_{m, 1 / 2} v \in \mathrm{H}^{1}\left(\mathbb{R}^{3}, \mathbb{C}\right),
$$

by scalar analogues of Lemmata 2.10 and 2.11 together with Lemma 2.5 we obtain

$$
\begin{aligned}
\left\langle v, R^{1} v\right\rangle & =\left\langle\Phi,(-\Delta)^{1 / 2} \Phi\right\rangle ; \\
\left\langle v, V_{0}(\cdot+\mathrm{i} / 2) R^{1} v\right\rangle & =\left\langle\Phi, r^{-1} \Phi\right\rangle .
\end{aligned}
$$

Thus (1.8) implies that (3.6) can be estimated from below by

$$
\left\langle\Phi,\left(L_{\lambda} a^{\lambda-1}(-\Delta)^{\lambda / 2}-a^{-1}\right) \Phi\right\rangle .
$$

Using the scalar version of Lemma 2.10 again and that $H_{0}^{\alpha_{0}}$ is the Friedrichs extension of $\widetilde{H}_{0}^{\alpha_{0}}$ we conclude (3.5).

The following description of the domains of $H_{l}^{\alpha}$ with $\alpha \leqslant \alpha_{l}$ follows analogously to Theorem 2 and Corollary 2 in 11 (see also Section 2.2.3 of [4), where the case $l=0$ is studied.

Lemma 3.3. Let $l \in \mathbb{N}_{0}$.

(i) For $\alpha<V_{l}^{-1}(\mathrm{i} / 2)$ the operator $\widetilde{H}_{l}^{\alpha}$ is self-adjoint, i.e. $\widetilde{H}_{l}^{\alpha}=H_{l}^{\alpha}$.

(ii) The operator $\widetilde{H}_{l}^{V_{l}^{-1}(\mathrm{i} / 2)}$ is essentially self-adjoint, i.e. the relation $\left(\widetilde{H}_{l}^{V_{l}^{-1}(\mathrm{i} / 2)}\right)^{*}=H_{l}^{V_{l}^{-1}(\mathrm{i} / 2)}$ holds.

(iii) For $\alpha \in\left(V_{l}^{-1}(\mathrm{i} / 2), \alpha_{l}\right]$ the Friedrichs extension $H_{l}^{\alpha}$ of $\widetilde{H}_{l}^{\alpha}$ is a restriction of

$$
\left(\widetilde{H}_{l}^{\alpha}\right)^{*}=R^{1}\left(1-\alpha V_{l}(\cdot-\mathrm{i} / 2)\right)
$$

to

$$
\mathfrak{D}\left(H_{l}^{\alpha}\right)=\mathfrak{D}^{1} \dot{+} \operatorname{span}\left\{\left(\cdot-\mathrm{i} / 2+\mathrm{i} \zeta_{l, \alpha}\right)^{-1}\right\},
$$

where $\zeta_{l, \alpha}$ is the unique solution of

$$
1-\alpha V_{l}\left(-\mathrm{i} \zeta_{l, \alpha}\right)=0
$$

in $(-1 / 2,0]$.

We now make a crucial observation concerning the functions (2.16) transformed in Lemma 2.12. The following lemma can be proved in the same way as Lemma 24 in 12 using Lemma 3.3

Lemma 3.4. Let $\nu \in(\sqrt{3} / 2,1]$. For $s= \pm 1 / 2$ the functions (2.20), (2.21) and (2.22) satisfy:

1. $\xi_{s}^{\nu}$ and $\eta_{s}^{\nu}$ belong to $\mathfrak{D}^{1}$;

2. $\chi_{s}^{\nu}$ belongs to $\mathfrak{D}\left(H_{0}^{\left(V_{0}\left(\mathrm{i} \Upsilon_{\nu}\right)\right)^{-1}}\right) \cap \mathfrak{D}\left(H_{1}^{\left(V_{1}\left(\mathrm{i} \Upsilon_{\nu}\right)\right)^{-1}}\right)$. 


\section{Channel-wise estimates}

We will now prove lower bounds on $\left|D_{l, s}^{\nu}\right|$ for $(l, m, s) \in \mathfrak{T}$ and $\nu \in[0,1]$. For $(\nu, l, s) \in(\sqrt{3} / 2,1] \times\{(0,1 / 2),(1,-1 / 2)\}$ the operator $\left(D_{l, s}^{\nu}\right)^{2}$ cannot be bounded from below by an operator unitarily equivalent to $R^{2}$, but for all other $(\nu, l, s)$ this is possible. Thus we distinguish two different types of channels.

Critical channels. For $\nu \in[0,1]$ and $s= \pm 1 / 2$ we introduce the $(2 \times 2)$-matrixvalued function on $\mathbb{R}$ :

$$
M_{s}^{\nu}:=\left(\begin{array}{cc}
-\nu V_{1 / 2-s}(\cdot+\mathrm{i} / 2) & 1 \\
1 & -\nu V_{1 / 2+s}(\cdot+\mathrm{i} / 2)
\end{array}\right) .
$$

Lemma 4.1. Let $\nu \in(\sqrt{3} / 2,1]$. For $s= \pm 1 / 2$ and any $\Psi \in \mathfrak{C}_{1 / 2-s, s}^{\nu}$ there exists a decomposition

$$
\mathcal{U}_{1 / 2-s, s} \Psi=\left(\begin{array}{l}
\zeta \\
v
\end{array}\right)+a \chi_{s}^{\nu}\left(\begin{array}{c}
1 \\
\nu V_{1 / 2-s}\left(\mathrm{i} \Upsilon_{\nu}\right)
\end{array}\right)
$$

with $\zeta, v \in \mathfrak{D}^{1}$ and $a \in \mathbb{C}$. Moreover, the representation

$$
\mathcal{U}_{1 / 2-s, s} D_{1 / 2-s, s}^{\nu} \Psi=M_{s}^{\nu}\left(\begin{array}{c}
R^{1} \zeta+a \chi_{s}^{\nu}(\cdot+\mathrm{i}) \\
R^{1} v+a \nu V_{1 / 2-s}\left(\mathrm{i} \Upsilon_{\nu}\right) \chi_{s}^{\nu}(\cdot+\mathrm{i})
\end{array}\right)
$$

holds.

Proof. The prove follows the same lines as the one of Lemma 24 in 12. The decomposition (4.2) follows from (2.15), Lemma 2.12, and Lemma 3.4. For any $(\varpi, \varsigma) \in \mathrm{C}_{0}^{\infty}\left(\mathbb{R}_{+}, \mathbb{C}^{2}\right)$ using (2.14), (2.19), Lemmata2.10 and2.11] Lemma2.5] and (3.7) we obtain

$$
\left\langle D_{1 / 2-s, s}^{\nu} \Psi,\left(\begin{array}{c}
\varpi \\
\varsigma
\end{array}\right)\right\rangle=\left\langle\mathcal{U}_{1 / 2-s, s}^{*} M_{s}^{\nu}\left(\begin{array}{c}
R^{1} \zeta+a \chi_{s}^{\nu}(\cdot+\mathrm{i}) \\
R^{1} v+a \nu V_{1 / 2-s}\left(\mathrm{i} \Upsilon_{\nu}\right) \chi_{s}^{\nu}(\cdot+\mathrm{i})
\end{array}\right),\left(\begin{array}{c}
\varpi \\
\varsigma
\end{array}\right)\right\rangle .
$$

By density of $\mathrm{C}_{0}^{\infty}\left(\mathbb{R}_{+}, \mathbb{C}^{2}\right)$ the claim follows.

The proof of the following lemma can be found in Appendix

Lemma 4.2. The map $\nu \mapsto \eta_{\nu}$ given by (1.5) is real-analytic and monotonously decreasing on $[0,1]$.

The next lemma is the most technically demanding.

Lemma 4.3. For $\nu \in[0,1]$ define the function

$$
K^{\nu}:=\left|1-\left(V_{0}\left(\mathrm{i} \Upsilon_{\nu}\right)\right)^{-1} V_{0}(\cdot+\mathrm{i} / 2)\right|^{2}
$$

on $\mathbb{R}$. Then for $s= \pm 1 / 2$ the maximal value of $\eta_{\nu}$ for which the lower bound

$$
\left(M_{s}^{\nu}\right)^{*} M_{s}^{\nu} \geqslant \eta_{\nu}^{2} K^{\nu} \otimes \mathbb{1}_{\mathbb{C}^{2}}
$$

holds point-wise on $\mathbb{R}$ is given by (1.5). 
Proof. It is enough to study the case of $M_{1 / 2}^{\nu}$ in (4.4), since the case of $M_{-1 / 2}^{\nu}$ follows immediately from the relation $M_{-1 / 2}^{\nu}=\sigma_{1} M_{1 / 2}^{\nu} \sigma_{1}$. For $\tau \in \mathbb{R}$ consider

$$
P(\tau):=\frac{\Gamma((3-2 \mathrm{i} \tau) / 4) \Gamma((1+2 \mathrm{i} \tau) / 4)}{\Gamma((3+2 \mathrm{i} \tau) / 4) \Gamma((1-2 \mathrm{i} \tau) / 4)} .
$$

For all $\tau \in \mathbb{R}$ we have

$$
|P(\tau)|=1
$$

and (see 5.4.5, 4.35.11, 4.35.16, 4.35.19, 4.28.4 and 4.28.6 in [1])

$$
P(\tau)=\operatorname{sech}(\pi \tau)-\mathrm{i} \tanh (\pi \tau) .
$$

By (2.8) we have

$$
V_{0}(\tau+\mathrm{i} / 2)=\frac{2}{1-2 \mathrm{i} \tau} P(\tau), \quad V_{1}(\tau-\mathrm{i} / 2)=\frac{2(1+2 \mathrm{i} \tau)}{(1-2 \mathrm{i} \tau)(3+2 \mathrm{i} \tau)} P(\tau) .
$$

Thus according to (4.1) for $\tau \in \mathbb{R}$ we have

$$
\left(M_{1 / 2}^{\nu}(\tau)\right)^{*} M_{1 / 2}^{\nu}(\tau)=\left(\begin{array}{cc}
1+\frac{4 \nu^{2}}{1+4 \tau^{2}} & \frac{-8 \nu(1-\mathrm{i} \tau) \overline{P(\tau)}}{(1+2 \mathrm{i} \tau)(3-2 \mathrm{i} \tau)} \\
\frac{-8 \nu(1+\mathrm{i} \tau) P(\tau)}{(1-2 \mathrm{i} \tau)(3+2 \mathrm{i} \tau)} & 1+\frac{4 \nu^{2}}{9+4 \tau^{2}}
\end{array}\right)
$$

In the following the function $z \cot z$ is analytically continued to $z=0$, i.e. $\left.z \cot z\right|_{z=0}:=1$. Representations (4.3), (4.5) and (4.6) imply

$$
K^{\nu}(\tau)=\frac{1+4 \tau^{2}+4 \Upsilon_{\nu}^{2} \cot ^{2}\left(\frac{\pi \Upsilon_{\nu}}{2}\right)-4 \Upsilon_{\nu} \cot \left(\frac{\pi \Upsilon_{\nu}}{2}\right)(\operatorname{sech}(\pi \tau)+2 \tau \tanh (\pi \tau))}{1+4 \tau^{2}} .
$$

Inequality (4.4) is equivalent to the non-negativity of the smallest eigenvalue of

$$
\left(M_{1 / 2}^{\nu}(\tau)\right)^{*} M_{1 / 2}^{\nu}(\tau)-\eta_{\nu}^{2} K^{\nu}(\tau) \otimes \mathbb{1}_{\mathbb{C}^{2}}
$$

Using (4.7) and (4.8) we find this eigenvalue to be given by

$$
\lambda(\tau)=1-\eta_{\nu}^{2} K^{\nu}(\tau)+\frac{4 \nu^{2}\left(5+4 \tau^{2}\right)-8 \nu\left(4 \nu^{2}+\left(1+\tau^{2}\right)\left(1+4 \tau^{2}\right)\left(9+4 \tau^{2}\right)\right)^{1 / 2}}{\left(1+4 \tau^{2}\right)\left(9+4 \tau^{2}\right)} .
$$

Now it is enough to prove the non-negativity of (4.9) with $\eta_{\nu}$ and $K^{\nu}$ given by (1.5) and (4.8), respectively. Using (4.8) and (1.5) we observe that the right hand side of (4.9) vanishes at $\tau=0$, i.e. the value of $\eta_{\nu}$ cannot be increased. 
Multiplying (4.9) by $\left(1+4 \tau^{2}\right)\left(9+4 \tau^{2}\right)$ and rewriting the result using (4.8) we conclude that it is enough to prove the non-negativity of

$$
\begin{aligned}
p(\tau, \nu) & :=\left(1-\eta_{\nu}^{2}\right)\left(1+4 \tau^{2}\right)\left(9+4 \tau^{2}\right)+4 \nu^{2}\left(5+4 \tau^{2}\right) \\
& +4 \Upsilon_{\nu} \eta_{\nu}^{2}\left(9+4 \tau^{2}\right)(\operatorname{sech}(\pi \tau)+2 \tau \tanh (\pi \tau)) \cot \left(\pi \Upsilon_{\nu} / 2\right) \\
& -4 \Upsilon_{\nu}^{2} \eta_{\nu}^{2}\left(9+4 \tau^{2}\right) \cot ^{2}\left(\pi \Upsilon_{\nu} / 2\right) \\
& -8 \nu\left(4 \nu^{2}+\left(1+\tau^{2}\right)\left(1+4 \tau^{2}\right)\left(9+4 \tau^{2}\right)\right)^{1 / 2}
\end{aligned}
$$

for all $(\tau, \nu) \in[0, \infty) \times[0,1]$.

Observing that

$$
\left(9+4 \tau^{2}\right)(1+2 \tau \sinh (\pi \tau))-\left(9+\left(4+18 \pi-9 \pi^{2} / 2\right) \tau^{2}\right) \cosh (\pi \tau) \geqslant 0
$$

holds for all $\tau \in \mathbb{R}$ by positivity of all the coefficients in the Taylor expansion at $\tau=0$, we conclude

$$
\left(9+4 \tau^{2}\right)(\operatorname{sech}(\pi \tau)+2 \tau \tanh (\pi \tau)) \geqslant 9+\left(4+18 \pi-9 \pi^{2} / 2\right) \tau^{2} .
$$

Thus to prove the non-negativity of (4.10) it suffices to establish the inequality

$$
p_{+}(\tau, \nu) \geqslant p_{-}(\tau, \nu)
$$

with

$$
\begin{aligned}
p_{+}(\tau, \nu) & :=\left(1-\eta_{\nu}^{2}\right)\left(1+4 \tau^{2}\right)\left(9+4 \tau^{2}\right)+4 \nu^{2}\left(5+4 \tau^{2}\right) \\
& +4 \Upsilon_{\nu} \eta_{\nu}^{2}\left(9+\left(4+18 \pi-9 \pi^{2} / 2\right) \tau^{2}\right) \cot \left(\pi \Upsilon_{\nu} / 2\right) \\
& -4 \Upsilon_{\nu}^{2} \eta_{\nu}^{2}\left(9+4 \tau^{2}\right) \cot ^{2}\left(\pi \Upsilon_{\nu} / 2\right)
\end{aligned}
$$

and

$$
p_{-}(\tau, \nu):=8 \nu\left(4 \nu^{2}+\left(1+\tau^{2}\right)\left(1+4 \tau^{2}\right)\left(9+4 \tau^{2}\right)\right)^{1 / 2} .
$$

For $x \in[0,1]$ we have

$$
x^{2} \cot ^{2}(\pi x / 2) \leqslant x \cot (\pi x / 2)<1,
$$

and, since Lemma 4.2 implies $\eta_{\nu} \leqslant \eta_{0}=1$, we get $p_{+}(\tau, \nu) \geqslant 0$. Thus (4.11) is equivalent to

$$
\left(p_{+}(\tau, \nu)\right)^{2}-\left(p_{-}(\tau, \nu)\right)^{2}=c_{2}(\nu) \tau^{2}+c_{4}(\nu) \tau^{4}+c_{6}(\nu) \tau^{6}+c_{8}(\nu) \tau^{8} \geqslant 0
$$

with

$$
\begin{aligned}
c_{2}(\nu) & :=\frac{16 \nu \sqrt{9+4 \nu^{2}}}{9}\left(72\left(5+2 \nu^{2}\right)-\frac{1764 \nu}{\sqrt{9+4 \nu^{2}}}+\left(\sqrt{9+4 \nu^{2}}-4 \nu\right)^{2}\right. \\
& \left.\times\left(-4+\frac{9 \pi^{2}(\pi-4)^{2}}{4(\pi-2)^{2}}-\left(\frac{3 \pi(4-\pi)}{2(\pi-2)}+\frac{3(\pi-2)}{1-2 \Upsilon_{\nu} \cot \left(\pi \Upsilon_{\nu} / 2\right)}\right)^{2}\right)\right), \\
c_{4}(\nu) & :=\frac{\left(c_{2}(\nu)+3136 \nu^{2}\right)^{2}}{256 \nu^{2}\left(9+4 \nu^{2}\right)}-3584 \nu^{2}+256 \nu \sqrt{9+4 \nu^{2}}\left(1-\eta_{\nu}^{2}\right), \\
c_{6}(\nu) & :=\frac{2\left(c_{2}(\nu)+3136 \nu^{2}\right)}{\nu \sqrt{9+4 \nu^{2}}}\left(1-\eta_{\nu}^{2}\right)-1024 \nu^{2}
\end{aligned}
$$


and

$$
c_{8}(\nu):=256\left(1-\eta_{\nu}^{2}\right)^{2} .
$$

In order to establish (4.12) it is enough to observe that for all $j=2,4,6,8$ the functions $c_{j}$ are non-negative on $[0,1]$. Taylor series expansions show that $\nu=0$ is a local minimum with value 0 for all of these functions, whereas $\nu=\sqrt{3} / 2$ is another local minimum with value 0 for $c_{2}$. B

A rigorous proof of the non-negativity of $c_{2}, c_{4}$ and $c_{6}$ can be found in Appendix

The following lemma is analogous to Lemma 28 in [12].

Lemma 4.4. The inequality

$$
\left(D_{1 / 2-s, s}^{\nu}\right)^{2} \geqslant \eta_{\nu}^{2}\left(\mathcal{U}_{1 / 2-s, s}^{*}\left(H_{0}^{\left(V_{0}\left(\mathrm{i} \Upsilon_{\nu}\right)\right)^{-1}} \otimes \mathbb{1}_{\mathbb{C}^{2}}\right) \mathcal{U}_{1 / 2-s, s}\right)^{2}
$$

holds for any $\nu \in[0,1]$ and $s= \pm 1 / 2$.

Proof. For $\nu \in(\sqrt{3} / 2,1]$ and arbitrary $\Psi \in \mathfrak{C}_{1 / 2-s, s}^{\nu}$ we use (4.2) to represent $\mathcal{U}_{s} \Psi$. Applying Lemmata 4.1, 4.3, 3.4 together with Lemma 3.3(iii) we get

$$
\begin{aligned}
& \left\|D_{1 / 2-s, s}^{\nu} \Psi\right\|^{2}=\left\|M_{s}^{\nu}\left(\begin{array}{c}
R^{1} \zeta+a \chi_{s}^{\nu}(\cdot+\mathrm{i}) \\
R^{1} v+a \nu V_{1 / 2-s}\left(\mathrm{i} \Upsilon_{\nu}\right) \chi_{s}^{\nu}(\cdot+\mathrm{i})
\end{array}\right)\right\|^{2} \\
& \geqslant \eta_{\nu}^{2}\left\|\left(1-\frac{V_{0}(\cdot+\mathrm{i} / 2)}{V_{0}\left(\mathrm{i} \Upsilon_{\nu}\right)}\right)\left(\begin{array}{c}
R^{1} \zeta+a \chi_{s}^{\nu}(\cdot+\mathrm{i}) \\
R^{1} v+a \nu V_{1 / 2-s}\left(\mathrm{i} \Upsilon_{\nu}\right) \chi_{s}^{\nu}(\cdot+\mathrm{i})
\end{array}\right)\right\|^{2} \\
& =\eta_{\nu}^{2} \| \mathcal{U}_{1 / 2-s, s}^{*}\left(H_{0}^{\left(V_{0}\left(\mathrm{i} \Upsilon_{\nu}\right)\right)^{-1}} \otimes \mathbb{1}_{\left.\mathbb{C}^{2}\right) \mathcal{U}_{1 / 2-s, s} \Psi \|^{2} .}\right.
\end{aligned}
$$

Since $\mathfrak{C}_{1 / 2-s, s}^{\nu}$ is an operator core for $D_{1 / 2-s, s}^{\nu}$, we conclude (4.15).

For $\nu \in[0, \sqrt{3} / 2]$, according to (2.15) we can do the same computation with $a:=0$ using parts $(i)$ and (ii) of Lemma 3.3 instead of part (iii).

\section{Non-critical channels.}

Lemma 4.5. Let $(l, m, s) \in \mathfrak{T}$ such that $(l, s) \notin\{(0,1 / 2),(1,-1 / 2)\}$. Then the operator inequalities

$$
\left(D_{l, s}^{\nu}\right)^{2} \geqslant \frac{\left(\left(225+4 \nu^{2}\right)^{1 / 2}-8 \nu\right)^{2}}{225}\left(\mathcal{U}_{l, s}^{*} R^{1} \mathcal{U}_{l, s}\right)^{2}
$$

hold for all $\nu \in[0,1]$.

Proof. Analogously to the proof of Lemma 29 in [12] we conclude that the inequality

$$
\left(D_{l, s}^{\nu}\right)^{2} \geqslant(1-b)\left(\mathcal{U}_{l, s}^{*} R^{1} \mathcal{U}_{l, s}\right)^{2}
$$


with $b \in[0,1]$ follows from the non-negativity of the function

$$
a_{\varkappa,-}^{\nu}(b, \tau):=\nu^{2}+b / 4+\varkappa^{2} b+\tau^{2} b-\left(4 \varkappa^{2} \nu^{2}+4 \nu^{2} \tau^{2}+\varkappa^{2} b^{2}\right)^{1 / 2},
$$

with $\varkappa:=2 s l+s+1 / 2 \in \mathbb{Z} \backslash\{-1,0,1\}$ for all $\tau \in \mathbb{R}$. Again as in the proof of Lemma 29 in [12, we observe that the function (4.16) is even in both $\varkappa$ and $\tau$ and monotonously increasing in $\varkappa$ for $\varkappa \geqslant 2, \tau \in \mathbb{R}$ provided $b \geqslant \nu / \sqrt{6}$. Furthermore, $a_{2,-}^{\nu}(b, \cdot)$ is monotonously increasing on $[0, \infty)$ provided $b \geqslant(\sqrt{5}-2)^{1 / 2} \nu$. It remains to find the smallest $b \in[0,1]$ satisfying the above assumptions together with $a_{2,-}^{\nu}(b, 0) \geqslant 0$.

\section{Proofs of the main theorems}

\subsection{Proof of Theorem 1.1}

In the same way as in the proof of Theorem 1 in [12, we obtain from Lemmata 2.7 3.1, $4.4,4.5$ and 2.10 the inequality (1.3) with

$$
C_{\nu}:=\min \left\{\eta_{\nu}\left(1-\frac{V_{0}(0)}{V_{0}\left(\mathrm{i} \Upsilon_{\nu}\right)}\right), \frac{1}{15}\left(\sqrt{225+4 \nu^{2}}-8 \nu\right)\right\} .
$$

Using (2.8) we observe the identity

$$
\eta_{\nu}\left(1-\frac{V_{0}(0)}{V_{0}\left(\mathrm{i} \Upsilon_{\nu}\right)}\right)=\frac{\pi}{12}\left(\sqrt{9+4 \nu^{2}}-4 \nu\right)+(1-\pi / 4) \eta_{\nu} .
$$

Hence by Lemma 4.2 and concavity of the square root we obtain

$$
\eta_{\nu}\left(1-\frac{V_{0}(0)}{V_{0}\left(\mathrm{i} \Upsilon_{\nu}\right)}\right) \leqslant \frac{\pi}{12}\left(3\left(1+2 \nu^{2} / 9\right)-4 \nu\right)+(1-\pi / 4)
$$

Estimating

$$
\frac{1}{15}\left(\sqrt{225+4 \nu^{2}}-8 \nu\right) \geqslant \frac{1}{15}(\sqrt{225}-8 \nu) \geqslant \frac{\pi}{12}\left(3\left(1+2 \nu^{2} / 9\right)-4 \nu\right)+(1-\pi / 4)
$$

for $\nu \in[0,1]$ and comparing to (5.2) we conclude that the minimum in (5.1) is always achieved at the first entry, i.e. (1.4) holds.

\subsection{Proof of Corollary 1.2}

For arbitrary $M>0$ the explicit solution to the eigenvalue problem (see e.g. (7.118) in [18) provides the lower bound

$$
\left(D^{\nu, M}\right)^{2} \geqslant M^{2}\left(1-\nu^{2}\right) .
$$

It implies, in its turn,

$$
\left(D^{\nu, M}\right)^{2}=\Upsilon_{\nu}^{2}\left(D^{\nu}\right)^{2}+\nu^{2}\left(D^{\nu, M / \nu^{2}}\right)^{2}+M^{2}\left(1-\nu^{-2}\right) \geqslant \Upsilon_{\nu}^{2}\left(D^{\nu}\right)^{2} .
$$


By operator monotonicity of the square root and Theorem 1.1 we conclude (1.6).

Taking a convex combination of (1.6) and the square root of (5.3), for any $\theta \in[0,1]$ we get

$$
\left|D^{\nu, M}\right| \geqslant \Upsilon_{\nu}\left(\theta C_{\nu}(-\Delta)^{1 / 2}+(1-\theta) M\right) .
$$

Computing

$$
\max _{\theta \in[0,1]} \inf _{p \in[0, \infty)} \frac{\theta C_{\nu} p+(1-\theta) M}{\sqrt{p^{2}+M^{2}}}
$$

we arrive at

$$
\left|D^{\nu, M}\right| \geqslant \frac{\Upsilon_{\nu} C_{\nu}}{1+C_{\nu}}\left|D^{0, M}\right| .
$$

Combining this with the estimate

$$
\left|D^{\nu, M}\right| \geqslant(1-2 \nu)\left|D^{0, M}\right|,
$$

which follows immediately from the Hardy inequality (see Lemma 1 in [3]), we obtain (1.7).

\subsection{Proof of Theorem 1.3}

The proof is analogous to the one of part 2 of Theorem $1 \mathrm{in} \mathrm{[12}$. It is enough to combine Lemmata $4.4,4.5,3.2$ with the inequality

$$
R^{1} \geqslant \lambda^{-\lambda}(1-\lambda)^{\lambda-1} a^{\lambda-1} R^{\lambda}-a^{-1}
$$

valid for all $a, R \in[0, \infty)$.

\subsection{Proof of Theorem 1.7}

The proof is analogous to the one of Part I of Theorem 2.5 in [13.

Let $\mathbf{j} \in\{-1,1\}^{2}$ be such that the conditions (1.11) and (1.12) are satisfied. For any $\psi \in P_{[0, \infty)}\left(D_{\left(j_{2}+1\right) / 2,-j_{2} / 2}^{1}\right) \mathfrak{D}\left(D_{\left(j_{2}+1\right) / 2,-j_{2} / 2}^{1}\right)$ we have

$$
\Psi:=\mathcal{A}^{*} \bigoplus_{(l, m, s) \in \mathfrak{T}} \delta_{l, j_{2} / 2+1 / 2} \delta_{m, j_{1} / 2} \delta_{s,-j_{2} / 2} \psi \in P_{+}^{1} \mathfrak{D}\left(D^{1}\right)
$$

and a calculation based upon (2.14), (2.11), (2.10), (2.1.25) and (2.1.26) in 4 delivers

$$
\left\langle\Psi, D^{1}(V) \Psi\right\rangle_{\mathrm{L}^{2}\left(\mathbb{R}^{3}, \mathbb{C}^{4}\right)}=\left\langle\psi,\left(D_{\left(j_{2}+1\right) / 2,-j_{2} / 2}^{1}-W_{\mathbf{j}} / 4 \pi\right) \psi\right\rangle_{\mathbf{L}^{2}\left(\mathbb{R}_{+}, \mathbb{C}^{2}\right)} .
$$

Now Part I of Theorem 2.3 in [13] guarantees the existence of $\psi$ such that (5.4) is negative. Hence by the minimax principle $D^{1}(V)$ has non-empty negative spectrum. According to Theorem 1.6(b) the negative spectrum of $D^{1}(V)$ can only consist of eigenvalues. 


\section{A Proof of Lemma 4.2}

Both $g(\nu):=\left(9+4 \nu^{2}\right)^{1 / 2}-4 \nu$ and $h(\nu):=3\left(1-2 \Upsilon_{\nu} \cot \left(\pi \Upsilon_{\nu} / 2\right)\right)$ (with the convention $\left.z \cot z\right|_{z=0}:=1$ ) are monotonously decreasing real-analytic functions of $\nu \in[0,1]$ taking value 3 at $\nu=0$ and having simple zeroes at $\nu=\sqrt{3} / 2$. Thus the real analyticity of $\eta_{\nu}$ follows from such of the numerator and denominator in (1.5) and the cancellation of zeroes. In the following it is enough to consider $\nu \in(0,1)$ and extend the results by continuity.

The monotonicity of $\nu \mapsto \eta_{\nu}$ follows from convexity of $g$ and concavity of $h$ together with the fact that both functions are monotonously decreasing and vanish at $\nu=\sqrt{3} / 2$. Indeed, then $|g(\nu)| /|\nu-\sqrt{3} / 2|$ is monotonously decreasing and $|h(\nu)| /|\nu-\sqrt{3} / 2|$ is monotonously increasing. Thus for every $\nu_{1}<\nu_{2} \in$ $(0,1) \backslash\{\sqrt{3} / 2\}$ we have

$$
\frac{g\left(\nu_{2}\right)}{h\left(\nu_{2}\right)}=\frac{\left|g\left(\nu_{2}\right)\right|}{\left|h\left(\nu_{2}\right)\right|} \leqslant \frac{\left|g\left(\nu_{1}\right)\right|}{\left|h\left(\nu_{1}\right)\right|}=\frac{g\left(\nu_{1}\right)}{h\left(\nu_{1}\right)} .
$$

The claimed convexity of $g$ and concavity of $h$ can be observed by studying their second derivatives. We have $g^{\prime \prime}(\nu)=36\left(9+4 \nu^{2}\right)^{-3 / 2}>0$ and $h^{\prime \prime}(\nu)=3\left(f_{1}(\nu)-\right.$ $\left.f_{2}(\nu)\right)$ with

$$
\begin{aligned}
f_{1}(\nu) & :=\frac{\nu^{2}\left(\pi \Upsilon_{\nu} \sin \left(\pi \Upsilon_{\nu} / 2\right)-\pi^{2} \Upsilon_{\nu}^{2} \cos \left(\pi \Upsilon_{\nu} / 2\right)+2 \cos \left(\pi \Upsilon_{\nu} / 2\right) \sin ^{2}\left(\pi \Upsilon_{\nu} / 2\right)\right)}{\Upsilon_{\nu}^{3} \sin ^{3}\left(\pi \Upsilon_{\nu} / 2\right)} \\
& <2
\end{aligned}
$$

and

$$
f_{2}(\nu):=\frac{\pi \Upsilon_{\nu}-\sin \left(\pi \Upsilon_{\nu}\right)}{\Upsilon_{\nu} \sin ^{2}\left(\pi \Upsilon_{\nu} / 2\right)}>2 .
$$

To justify (A.1), for $z \in[0, \pi / 2]$ we can combine the elementary estimates

$$
\begin{gathered}
2 z \sin z \leqslant 2 z^{2}-\frac{z^{4}}{3}+\frac{z^{6}}{60}, \\
-4 z^{2} \cos z \leqslant-4 z^{2}+2 z^{4}-\frac{z^{6}}{6}+\frac{z^{8}}{180}, \\
2(\cos z) \sin ^{2} z=(\cos z)(1-\cos (2 z)) \leqslant\left(1-\frac{z^{2}}{2}+\frac{z^{4}}{24}\right)\left(2 z^{2}-\frac{2 z^{4}}{3}+\frac{4 z^{6}}{45}\right)
\end{gathered}
$$

which follow from the Taylor expansions of $\sin z$ and $\cos z$ at $z=0$ to conclude

$$
\begin{aligned}
2 z \sin z-4 z^{2} \cos z+2(\cos z) \sin ^{2} z & \leqslant \frac{16}{45} z^{6}-\frac{z^{8}}{15}+\frac{z^{10}}{270} \\
& \leqslant \frac{16}{45} z^{6}+\left(-\frac{1}{15}+\frac{\pi^{2}}{4 \cdot 270}\right) z^{8} \leqslant \frac{16}{45} z^{6} .
\end{aligned}
$$


Analogously, for $z \in[0, \pi / 2]$ we get

$$
\sin ^{3} z \geqslant\left(z-\frac{z^{3}}{6}\right)^{3} \geqslant z^{3}-\frac{z^{5}}{2}+\left(\frac{1}{12}-\frac{\pi^{2}}{4 \cdot 216}\right) z^{7} .
$$

Multiplying (A.1) by the denominator and applying the inequalities A.3 and (A.4) we conclude its validity. Inequality (A.2) for $z \in[0, \pi / 2]$ follows analogously from

$$
\begin{aligned}
2 z-\sin (2 z) & \geqslant \frac{4 z^{3}}{3}-\frac{4 z^{5}}{15} \quad \text { and } \\
\sin ^{2} z=\frac{1-\cos (2 z)}{2} & \leqslant z^{2}-\frac{z^{4}}{3}+\frac{2 z^{6}}{45} .
\end{aligned}
$$

\section{B Remainder of the proof of Lemma 4.3: the non- negativity of $c_{2}, c_{4}$ and $c_{6}$}

We first prove appropriate estimates on the function $z \mapsto z \cot z$.

\section{Lemma B.1.}

1. For $n \in \mathbb{N}$ let

$$
F_{n}(\nu):=\sum_{k=1}^{n}(-1)^{k+1}\left(\sum_{j=1}^{\infty} \frac{16 j^{2}}{\pi\left(4 j^{2}-1\right)^{k+1}}\right) \nu^{2 k} .
$$

Then for all $\nu \in[0,1]$ and $n \in \mathbb{N}$ the inequalities

$$
F_{2 n}(\nu) \leqslant \Upsilon_{\nu} \cot \left(\pi \Upsilon_{\nu} / 2\right) \leqslant F_{2 n-1}(\nu)
$$

hold.

2. For $n \in \mathbb{N}$ let

$$
S_{n}:=\frac{2^{2 n+2}}{\pi} \sum_{k=0}^{\infty} \frac{(-1)^{k}}{(2 k+1)^{2 n+1}}, \quad \text { and } \quad T_{n}:=\frac{2^{2 n+1}}{\pi} \sum_{k=0}^{\infty} \frac{1}{(2 k+1)^{2 n}} .
$$

Then for all $x \in(-1 / 2,0]$ and $n \in \mathbb{N}$ the inequality

$$
\left(\frac{1}{2}+x\right) \cot \left(\frac{\pi(1 / 2+x)}{2}\right) \geqslant \frac{1}{2}+\sum_{j=1}^{n}\left(\frac{S_{j}}{2}-T_{j}\right) x^{2 j}+\left(S_{j-1}-\frac{T_{j}}{2}\right) x^{2 j-1}
$$

holds.

3. For all $x \in[0,1 / 2)$ and $l, k \in \mathbb{N}$ the inequality

$$
\left(\frac{1}{2}+x\right) \cot \left(\frac{\pi\left(\frac{1}{2}+x\right)}{2}\right) \leqslant \frac{1}{2}+\sum_{j=1}^{l}\left(\frac{S_{j}}{2}-T_{j}\right) x^{2 j}+\sum_{j=1}^{k}\left(S_{j-1}-\frac{T_{j}}{2}\right) x^{2 j-1}
$$

holds true. 
Proof. 1. Applying (1.2), the series representation 4.22.3 in [1] and using the geometric series we arrive at

$$
\Upsilon_{\nu} \cot \left(\pi \Upsilon_{\nu} / 2\right)=\frac{2}{\pi}-\frac{4}{\pi} \sum_{n=1}^{\infty} \frac{1}{4 n^{2}-1}+\sum_{k=1}^{\infty}(-1)^{k+1}\left(\sum_{n=1}^{\infty} \frac{16 n^{2}}{\pi\left(4 n^{2}-1\right)^{1+k}}\right) \nu^{2 k}
$$

By setting $\nu:=0$ in (B.5) we obtain

$$
\frac{2}{\pi}-\frac{4}{\pi} \sum_{n=1}^{\infty} \frac{1}{4 n^{2}-1}=0
$$

It remains to observe that the last series in (B.5) is alternating with monotonously decreasing absolute values of the terms.

2, 3. By 4.19.5, 24.8.4 and 24.2.9 in [1] we obtain

$$
\sec (\pi x)=\sum_{n=0}^{\infty} S_{n} x^{2 n} \quad \text { for }|x|<1 / 2 .
$$

By 4.19.3, 25.6.2, 24.2.2 and 25.2.2 in [1] we have

$$
\tan (\pi x)=\sum_{n=1}^{\infty} T_{n} x^{2 n-1} \quad \text { for }|x|<1 / 2
$$

Applying the trigonometric identity

$$
\cot (\pi(1 / 2+x) / 2)=\sec (\pi x)-\tan (\pi x)
$$

we arrive at

$$
(1 / 2+x) \cot (\pi(1 / 2+x) / 2)=\frac{1}{2}+\sum_{j=1}^{\infty}\left(\left(\frac{S_{j}}{2}-T_{j}\right) x+\left(S_{j-1}-\frac{T_{j}}{2}\right)\right) x^{2 j-1}
$$

Since (B.6) and (B.7) imply

$$
\frac{S_{j}}{2}-T_{j}<0 \quad \text { and } \quad S_{j-1}-\frac{T_{j}}{2}<0 \quad \text { for all } j \in \mathbb{N},
$$

(B.4) with $x \in[0,1 / 2)$ follows from (B.8).

For $x \in(-1 / 2,0]$ using (B.9) and (B.2) we conclude for $j \in \mathbb{N}$

$$
\begin{aligned}
& \left(\frac{S_{j}}{2}-T_{j}\right) x+\left(S_{j-1}-\frac{T_{j}}{2}\right) \leqslant \frac{1}{2}\left(T_{j}-\frac{S_{j}}{2}\right)+S_{j-1}-\frac{T_{j}}{2} \\
& =S_{j-1}-\frac{S_{j}}{4}=\frac{2^{2 j}}{\pi}\left(\sum_{k=1}^{\infty}(-1)^{k} \frac{(2 k+1)^{2}-1}{(2 k+1)^{2 j+1}}\right)<0 .
\end{aligned}
$$

Together with (B.8) and $x^{2 j-1} \leqslant 0$ this gives the lower bound (B.3). 
Non-negativity of $c_{2}$ for $\nu \in[0,3 / 5]$. Let $b_{1}(\nu)$ be given by the right hand side of (4.13), but with $\pi \nu^{2} / 2$ instead of $2 \Upsilon_{\nu} \cot \left(\pi \Upsilon_{\nu} / 2\right)$. The inequality

$$
1-2 \Upsilon_{\nu} \cot \left(\pi \Upsilon_{\nu} / 2\right) \geqslant 1-\pi \nu^{2} / 2
$$

which follows from (B.1) implies that $b_{1}(\nu) \leqslant c_{2}(\nu)$ holds for all $\nu$ for which the right hand side of (B.11) remains positive, thus for all $\nu \in[0,3 / 5]$. Hence the non-negativity of $c_{2}(\nu)$ follows from the non-negativity of

$$
\frac{9\left(\pi \nu^{2}-2\right)^{2}}{32 \nu^{2}} b_{1}(\nu)=p_{1}(\nu)+q_{1}(\nu)
$$

where

$$
\begin{aligned}
p_{1}(\nu) & :=2232+\left(2560+2952 \pi-2592 \pi^{2}+648 \pi^{3}\right) \nu^{2} \\
& +\left(288 \pi^{3}-256 \pi-1890 \pi^{2}\right) \nu^{4}+64 \pi^{2} \nu^{6} \geqslant 0 \text { for all } \nu \in[0,3 / 5]
\end{aligned}
$$

and

$$
\begin{aligned}
q_{1}(\nu) & :=\left(\left(324 \pi^{2}-1312-648 \pi-81 \pi^{3}\right) \nu\right. \\
& \left.+\left(882 \pi^{2}-128 \pi-180 \pi^{3}\right) \nu^{3}+32 \pi^{2} \nu^{5}\right) \sqrt{9+4 \nu^{2}}
\end{aligned}
$$

To establish the non-negativity of (B.12) it is thus enough to observe the nonnegativity of

$$
p_{2}(\nu):=p_{1}^{2}(\nu)-q_{1}^{2}(\nu)
$$

which is a polynomial of degree 5 in $\nu^{2}$. Such non-negativity for $\nu \in[0,3 / 5]$ follows immediately from the fact that

$$
p_{3}(y):=p_{2}(\sqrt{9 / 25-y})
$$

is a polynomial with positive coefficients and is thus positive for all $y \in[0,9 / 25]$.

Non-negativity of $c_{2}$ for $\nu \in[3 / 5,1]$. Substituting $\nu:=\sqrt{1-(1 / 2+x)^{2}}$ with $x \in[-1 / 2,1 / 2]$ and introducing

$$
r_{1}(x):=\sqrt{12-4 x-4 x^{2}} \text { and } r_{2}(x):=\sqrt{3-4 x-4 x^{2}}
$$

we conclude that the non-negativity of $c_{2}(\nu)$ for $\nu \in[0,1]$ is equivalent to the non-negativity of

$$
\begin{aligned}
& d_{2}(x):=\frac{9 c_{2}\left(\sqrt{1-(1 / 2+x)^{2}}\right)}{16 \sqrt{1-(1 / 2+x)^{2}}} \\
& =r_{1}(x)\left(36\left(13-4 x-4 x^{2}\right)-882 \frac{r_{2}(x)}{r_{1}(x)}+\left(r_{1}(x)-2 r_{2}(x)\right)^{2}\right. \\
& \left.\times\left(\frac{9 \pi^{2}(4-\pi)^{2}}{4(\pi-2)^{2}}-4-\left(\frac{3 \pi(4-\pi)}{2(\pi-2)}+\frac{3(\pi-2)}{1-2(1 / 2+x) \cot (\pi(1 / 2+x) / 2)}\right)^{2}\right)\right)
\end{aligned}
$$


for $x \in[-1 / 2,1 / 2]$. Introducing for $x \in[-1 / 2,1 / 2]$ the functions

$$
h(x):=1+(2-\pi) x+\left(\frac{\pi^{2}}{2}-2 \pi\right) x^{2}+\left(\pi^{2}-\frac{\pi^{3}}{3}\right) x^{3}+\left(\frac{5 \pi^{4}}{24}-\frac{2 \pi^{3}}{3}\right) x^{4}
$$

and

$$
l(x):=2(1 / 2+x) \cot (\pi(1 / 2+x) / 2)
$$

by Lemma B.13 and (B.9) we obtain

$$
1-l(x) \geqslant 1-h(x) \geqslant 0 \text { for } x \in[0,1 / 2] .
$$

On the other hand, Lemma B.12, (B.10) and the monotonicity of $y \mapsto y \cot y$ on $[0, \pi / 2]$ imply

$$
1-l(-1 / 2) \leqslant 1-l(x) \leqslant 1-h(x) \leqslant 0 \text { for } x \in[-1 / 2,0] .
$$

Combining (B.13) and (B.14) we obtain

$$
\left(\frac{3 \pi(4-\pi)}{2(\pi-2)}+\frac{3(\pi-2)}{1-l(x)}\right)^{2} \leqslant\left(\frac{3 \pi(4-\pi)}{2(\pi-2)}+\frac{3(\pi-2)}{1-h(x)}\right)^{2}
$$

and thus

$$
\begin{aligned}
b_{2}(x) & :=r_{1}(x)\left(36\left(13-4 x-4 x^{2}\right)-882 \frac{r_{2}(x)}{r_{1}(x)}+\left(r_{1}(x)-2 r_{2}(x)\right)^{2}\right. \\
& \times\left(\frac{9 \pi^{2}(4-\pi)^{2}}{4(\pi-2)^{2}}-4-\left(\frac{3 \pi(4-\pi)}{2(\pi-2)}\right.\right. \\
& \left.\left.\left.+\frac{3(\pi-2)}{(\pi-2) x+\left(2 \pi-\frac{\pi^{2}}{2}\right) x^{2}+\left(\frac{\pi^{3}}{3}-\pi^{2}\right) x^{3}+\left(\frac{2 \pi^{3}}{3}-\frac{5 \pi^{4}}{24}\right) x^{4}}\right)^{2}\right)\right)
\end{aligned}
$$

is a lower bound on $d_{2}(x)$ for all $x \in[-1 / 2,1 / 2]$. To prove the non-negativity of $c_{2}(\nu)$ for $\nu \in[3 / 5,1]$ it is thus enough to prove the non-negativity of $b_{2}(x)$ for $x \in[-1 / 2,3 / 10]$, or, equivalently, the non-negativity of

$$
\begin{aligned}
g_{2}(y) & :=\frac{1}{4}(1-2 y)^{2}\left(384-5 \pi^{4}+\left(30 \pi^{4}-384 \pi-96 \pi^{2}-32 \pi^{3}\right) y\right. \\
& \left.+\left(192 \pi^{2}+128 \pi^{3}-60 \pi^{4}\right) y^{2}+\left(40 \pi^{4}-128 \pi^{3}\right) y^{3}\right)^{2} b_{2}(y-1 / 2)
\end{aligned}
$$

for $y \in[0,4 / 5]$. Expanding $g_{2}$ we obtain the representation

$$
g_{2}(y)=p_{4}(y) \sqrt{1-y^{2}}+p_{5}(y) \sqrt{13-4 y^{2}}
$$

with polynomials $p_{4}$ and $p_{5}$ of order 10 . We claim that

$$
p_{4}(y)=\sum_{k=0}^{10} v_{k} y^{k}
$$


is positive for all $y \in[0,4 / 5]$. Indeed, a positive lower bound on $p_{4}$ can be obtained by first replacing the positive coefficients $v_{5}, v_{7}$ and $v_{9}$ by zero, then for $k=3,4,6,8,10$ (for which $v_{k}$ are negative) estimating $v_{k} y^{k}$ from below by $(4 / 5)^{k-2} v_{k} y^{2}$, and estimating $v_{1} y$ from below by $4 v_{1} / 5$ (since $v_{1}$ is negative). As a result we obtain the estimate

$$
p_{4}(y) \geqslant \widetilde{v}_{0}+\widetilde{v}_{2} y^{2}>0 \quad \text { for } y \in[0,4 / 5]
$$

with positive $\widetilde{v}_{0}$ and $\widetilde{v}_{2}$. Thus to obtain the non-negativity of (B.15) on $[0,4 / 5]$ it suffices to prove positivity of the polynomial

$$
p_{6}(y):=\frac{p_{4}^{2}(y)\left(1-y^{2}\right)-p_{5}^{2}(y)\left(13-4 y^{2}\right)}{81(1-2 y)^{4}}=\sum_{k=0}^{16} w_{k} y^{k}
$$

of degree 16 on this interval. To get a positive lower bound on $p_{6}(y)$ for $y \in[0,4 / 5]$ we can replace the positive coefficients at $w_{13}$ and $w_{15}$ by zero. For all other $k \geqslant 7$ we have $w_{k}<0$ and estimate $w_{k} y^{k} \geqslant(4 / 5)^{k-6} w_{k} y^{6}$. For $k \in\{1, \ldots, 5\}$ we have $w_{k}>0$ and estimate $w_{k} y^{k} \geqslant w_{k} y^{6}$. As a result we obtain $p_{6}(y) \geqslant w_{0}+\widetilde{w}_{6} y^{6}$ with $w_{0}, \widetilde{w}_{6}>0$.

Non-negativity of $c_{4}(\nu)$ for $\nu \in[0,1]$. By the non-negativity of $c_{2}$ we obtain the lower bound

$$
16 \nu\left(-224 \nu+\frac{2401 \nu}{9+4 \nu^{2}}+16 \sqrt{9+4 \nu^{2}}\left(1-\eta_{\nu}^{2}\right)\right)
$$

on (4.14). By Lemma 4.2 the last term is non-negative and monotonously growing. Since the sum of the first two terms is non-negative for $\nu \in[0, \sqrt{55 / 128}]$, it is enough to establish the positivity of B.16 for $\nu \in[\sqrt{55 / 128}, 1]$. By concavity we can estimate

$$
-224 \nu+\frac{2401 \nu}{9+4 \nu^{2}} \geqslant \frac{7}{26}(16+\sqrt{110})(\sqrt{110}-16 \nu)
$$

(the right hand side is a linear interpolation between the values at $\nu=\sqrt{55 / 128}$ and $\nu=1$ ) and, by convexity,

$$
\sqrt{9+4 \nu^{2}} \geqslant \frac{9+4 \nu}{\sqrt{13}}
$$

(the right hand side is the tangent line at $\nu=1)$. Substituting (B.17) and (B.18) into (B.16) and using $\eta_{\nu} \leqslant \eta_{\sqrt{55 / 128}}$ for $\nu \in[\sqrt{55 / 128}, 1]$ we get a lower bound

$$
16 \nu\left(\frac{7}{26}(16+\sqrt{110})(\sqrt{110}-16 \nu)+\frac{16(9+4 \nu)}{\sqrt{13}}\left(1-\eta_{\sqrt{55 / 128}}^{2}\right)\right)
$$

on (4.14), where the last factor is a decreasing linear function positive for $\nu \leqslant 1$. 
Non-negativity of $c_{6}(\nu)$ for $\nu \in[0,1]$. We first observe that by the nonnegativity of $c_{2}$ we have a lower bound

$$
c_{6}(\nu) \geqslant \frac{6272 \nu\left(1-\eta_{\nu}^{2}\right)}{\sqrt{4 \nu^{2}+9}}-1024 \nu^{2} .
$$

Its non-negativity is equivalent to

$$
1-\eta_{\nu}^{2} \geqslant(8 / 49) \nu \sqrt{4 \nu^{2}+9},
$$

where by Lemma 4.2 both sides are monotonously growing. Due to

$$
1-\eta_{1 / 2}^{2}>(8 / 49) \sqrt{13}
$$

it remains to establish (B.19) for $\nu \in[0,1 / 2]$. For this we substitute (1.5) and the estimate (B.11) to obtain that (B.19) follows from

$$
\left(1280+288 \pi \nu^{2}-72 \pi^{2} \nu^{4}\right) \sqrt{4 \nu^{2}+9} \geqslant 441 \pi^{2} \nu^{3}-(1764 \pi+3920) \nu .
$$

Since the left hand side is clearly positive for $\nu \in[0,1 / 2]$, (B.20) follows from the non-negativity of the polynomial

$$
\begin{aligned}
p_{7}(\nu) & :=\left(1280+288 \pi \nu^{2}-72 \pi^{2} \nu^{4}\right)^{2}\left(4 \nu^{2}+9\right)-\left(441 \pi^{2} \nu^{2}-1764 \pi-3920\right)^{2} \nu^{2} \\
& =: \sum_{k=0}^{5} a_{2 k} \nu^{2 k} .
\end{aligned}
$$

To obtain a lower bound on $p_{7}(\nu)$ for $\nu \in[0,1 / 2]$ we use $a_{10} \geqslant 0$ and for $a_{8}, a_{6}<0$ apply the estimates $a_{8} \nu^{8} \geqslant(1 / 2)^{4} a_{8} \nu^{4}, a_{6} \nu^{6} \geqslant(1 / 2)^{2} a_{6} \nu^{4}$ obtaining

$$
p_{7}(\nu) \geqslant a_{0}+a_{2} \nu^{2}+\left(a_{4}+(1 / 2)^{4} a_{8}+(1 / 2)^{2} a_{6}\right) \nu^{4},
$$

where the right hand side is positive.

\section{References}

[1] NIST digital library of mathematical functions. http://dlmf.nist.gov/, Release 1.0.10 of 2015-08-07. Online companion to [15].

[2] R. A. Adams and J. J. F. Fournier. Sobolev spaces, volume 140 of Pure and Applied Mathematics (Amsterdam). Elsevier/Academic Press, Amsterdam, second edition, 2003.

[3] V. Bach, J.-M. Barbaroux, B. Helffer, and H. Siedentop. On the Stability of the Relativistic Electron-Positron Field. Communications in Mathematical Physics, 201(2):445-460, 1999.

[4] A. A. Balinsky and W. D. Evans. Spectral analysis of relativistic operators. Imperial College Press, London, 2011. 
[5] M. S. Birman and M. Z. Solomjak. Spectral theory of selfadjoint operators in Hilbert space. Mathematics and its Applications (Soviet Series). D. Reidel Publishing Co., Dordrecht, 1987.

[6] R. Brummelhuis, N. Röhrl, and H. Siedentop. Stability of the relativistic electron-positron field of atoms in Hartree-Fock approximation: Heavy elements. Doc. Math., J, 1505(6):1-8, 2001.

[7] M. J. Esteban and M. Loss. Self-adjointness for Dirac operators via HardyDirac inequalities. J. Math. Phys., 48(11):112107, 8, 2007.

[8] R. L. Frank. A simple proof of Hardy-Lieb-Thirring inequalities. Comm. Math. Phys., 290(2):789-800, 2009.

[9] R. L. Frank. Cwikel's theorem and the CLR inequality. J. Spectr. Theory, 4(1):1-21, 2014.

[10] K. Friedrichs. Spektraltheorie halbbeschränkter Operatoren und Anwendung auf die Spektralzerlegung von Differentialoperatoren. Mathematische Annalen, 109(1):465-487, 1934.

[11] A. Le Yaouanc, L. Oliver, and J.-C. Raynal. The Hamiltonian $\left(p^{2}+m^{2}\right)^{1 / 2}-$ $\alpha / r$ near the critical value $\alpha_{c}=2 / \pi$. Journal of Mathematical Physics, 38(8):3997-4012, 1997.

[12] S. Morozov and D. Müller. Lieb-Thirring and Cwickel-Lieb-Rozenblum inequalities for perturbed graphene with a Coulomb impurity. Preprint arXiv:1603.01485, 2016.

[13] S. Morozov and D. Müller. On the virtual levels of positively projected massless coulomb-dirac operators. arXiv preprint arXiv:1607.08902, 2016.

[14] D. Müller. Minimax principles, Hardy-Dirac inequalities and operator cores for two and three dimensional Coulomb-Dirac operators. Documenta Mathematica, 21:1151-1169, 2016.

[15] F. W. J. Olver, D. W. Lozier, R. F. Boisvert, and C. W. Clark, editors. NIST Handbook of Mathematical Functions. Cambridge University Press, New York, NY, 2010. Print companion to [1].

[16] J. Rauch. Partial differential equations, volume 128 of Graduate Texts in Mathematics. Springer-Verlag, New York, 1991.

[17] J. P. Solovej, T. Ø. Sørensen, and W. L. Spitzer. Relativistic Scott correction for atoms and molecules. Comm. Pure Appl. Math., 63(1):39-118, 2010.

[18] B. Thaller. The Dirac equation. Texts and Monographs in Physics. SpringerVerlag, Berlin, 1992. 